\title{
Intraflagellar transport-A deficiency attenuates ADPKD in a renal tubular- and maturation-
}

\section{dependent manner}

Wei Wang ${ }^{1}$, Luciane M. Silva ${ }^{1}$, Henry H. Wang ${ }^{1}$, Matthew A. Kavanaugh ${ }^{1}$, Tana S. Pottorf ${ }^{1}$, Bailey A. Allard $^{1}$, Damon T. Jacobs ${ }^{1}$, Rouchen Dong ${ }^{1}$, Joseph T. Cornelius ${ }^{1}$, Aakriti Chaturvedi ${ }^{1}$, Michele T. Pritchard $^{2}$, Madhulika Sharma ${ }^{3}$, Chad Slawson ${ }^{4}$, Darren P. Wallace ${ }^{3}$, James P. Calvet ${ }^{4}$, Pamela V. $\operatorname{Tran}^{1}$

${ }^{1}$ Dept. of Anatomy and Cell Biology, The Jared Grantham Kidney Institute, University of Kansas Medical Center, Kansas City, KS

${ }^{2}$ Dept. of Pharmacology, Toxicology and Therapeutics, The Jared Grantham Kidney Institute, University of Kansas Medical Center, Kansas City, KS

${ }^{3}$ Dept. of Internal Medicine, The Jared Grantham Kidney Institute, University of Kansas Medical Center, Kansas City, KS

${ }^{4}$ Dept. of Biochemistry and Molecular Biology, The Jared Grantham Kidney Institute, University of Kansas Medical Center, Kansas City, KS

Correspondence should be addressed to:

Pamela V. Tran

Department of Anatomy and Cell Biology and

The Jared Grantham Kidney Institute

University of Kansas Medical Center

3901 Rainbow Blvd., MS \#3038

Kansas City, KS 66160

Tel: 913-945-7325

Fax: 913-588-2710

E-mail: ptran@kumc.edu 


\begin{abstract}
Primary cilia are sensory organelles built and maintained by intraflagellar transport (IFT) multiprotein complexes. Deletion of different IFT-B genes attenuates polycystic kidney disease (PKD) severity in juvenile and adult Autosomal Dominant (AD) PKD mouse models, while deletion of an IFTA adaptor, Tulp3, attenuates PKD severity in adult mice only. These studies indicate that dysfunction of specific cilia components has potential therapeutic value. To broaden our understanding of cilia dysfunction and its therapeutic potential, here we investigate the impact of global deletion of an IFT-A gene, Thm1, in juvenile and adult ADPKD mouse models. Both juvenile and adult models exhibited increased kidney weight:body weight $(\mathrm{KW} / \mathrm{BW})$ ratios, renal cysts, inflammation, lengthened renal cilia, and increased levels of the nutrient sensor, O-linked $\beta$-N-acetylglucosamine (O-GlcNAc). Thm 1 deletion in juvenile ADPKD mice reduced KW/BW ratios and cortical collecting duct cystogenesis, but increased proximal tubular and glomerular dilations and did not reduce inflammation, cilia lengths, and O-GlcNAc signaling. In contrast, Thm 1 deletion in adult ADPKD mice markedly attenuated renal cystogenesis, inflammation, cilia lengths, and O-GlcNAc. Thus, unlike IFT-B genes, the role of Thm1 deletion in ADPKD mouse models is development-specific. Unlike an IFT-A adaptor gene, deleting Thm 1 in juvenile ADPKD mice is partially ameliorative. Our studies suggest that different microenvironmental factors found in distinct nephron segments and between developing and mature kidneys modify ciliary homeostasis and ADPKD pathobiology. Further, elevated levels of O-GlcNAc, which regulates cellular metabolism and ciliogenesis, may be a novel feature and critical regulator of certain key ADPKD pathological processes.
\end{abstract}

Keywords: Ttc21B, IFT139, ciliopathy, early-onset, late-onset, renal cystic disease 


\section{Introduction}

Autosomal Dominant Polycystic Kidney Disease (ADPKD) is among the most common, fatal monogenetic diseases, affecting approximately 1:1000 individuals worldwide ${ }^{1}$. ADPKD is characterized by the growth of large fluid-filled renal cysts, which cause injury and fibrosis and can lead to end-stage renal disease by the $6^{\text {th }}$ decade of life. Tolvaptan is the only FDA-approved therapy, but has variable effectiveness and aquaresis side effects ${ }^{2,3}$. Thus, the need to discover additional underlying disease mechanisms and design new therapeutic strategies continues.

Primary cilia are small, antenna-like sensory organelles that play an important role in ADPKD pathobiology via mechanisms that remain unclear. ADPKD is caused by mutations in PKD1 or PKD2, which encode polycystin 1 (PC1) and polycystin 2 (PC2), respectively ${ }^{4}$ PC1 and PC2 form an ionchannel receptor complex that functions at the primary cilium. While PC1 and PC2 also localize to other subcellular compartments, analyses of human ADPKD primary renal epithelial cells, of mouse models harboring human ADPKD mutations, and of an ethylnitrosourea (ENU)-induced $P k d 2$ mouse mutation that causes ciliary exclusion of PC2, indicate that deficiency of polycystins from the cilium is sufficient to cause $\mathrm{ADPKD}^{5-7}$.

Primary cilia are synthesized and maintained via intraflagellar transport (IFT), which is the bidirectional transport of protein cargo along a microtubular axoneme. Two multiprotein complexes mediate IFT. The IFT-B complex interacts with the kinesin motor and mediates anterograde IFT, while the IFT-A complex together with cytoplasmic dynein mediates retrograde IFT. IFT-A proteins are also required for ciliary import of membrane and signaling molecules ${ }^{8-10}$. Additionally, an IFT-A adaptor, TULP3, binds to the IFT-A complex and brings in certain G-protein signaling molecules. In mice, deletion of Ift-A or $-B$ genes or of Tulp3, either perinatally or in the embryonic kidney results in renal cystic disease ${ }^{11-13}$. However, these mutants differ from ADPKD models in manifesting generally smaller renal cysts and greater fibrosis relative to cyst size ${ }^{14,15}$. Additionally, $I f t-A$ and $-B$ mutants differ in cilia phenotype - often shortened and absent cilia, respectively - and can also show opposing signaling 
phenotypes, reflecting the differing functions of IFT-A and $-\mathrm{B}^{12,16-18}$. Intriguingly, deletion of Ift-B genes, Kif $3 a$, Ift 20 and Ift 88 in juvenile and adult $P k d 1$ or $P k d 2$ conditional knock-out (cko) mice reduces PKD severity ${ }^{19-22}$, while deletion of Tulp3 attenuates PKD in adult mice only. Collectively, these studies indicate that a component of cilia dysfunction has potential therapeutic value.

The role of IFT-A deficiency in ADPKD has not been reported. THM1 (TPR-containing Hedgehog modulator 1; also termed TTTC21B) is an ortholog of Chlamydomonas IFT139, an IFT-A gene $^{16}$. Causative and modifying mutations in THMI have been identified in patients with nephronophthisis, Bardet Biedl syndrome, Meckel syndrome, Jeune syndrome and renal agenesis ${ }^{14}$. Characteristic of IFT-A mutation, deletion of Thm1 impairs retrograde IFT, causing accumulation of proteins in bulb-like distal tips of shortened primary cilia ${ }^{16}$. Thm 1 loss also impairs cilia entry of membrane-associated proteins, delays and reduces ciliogenesis, and promotes serum-induced cilia loss ${ }^{23}$. In mice, $T h m 1$ deletion recapitulates many of the clinical manifestations of ciliopathies ${ }^{16,24,25}$. Perinatal global deletion of Thm 1 results in renal cystic disease ${ }^{24}$, while deletion of Thm 1 in adult mice does not result in a renal phenotype by 3 months of age, consistent with the developmental time-frame that determines whether loss of a cystogenic gene will cause rapid- or slow-progressing renal cystic disease ${ }^{26}$. To expand on the role of ciliary dysfunction in ADPKD, here we investigate the role of Thm1/ IFT-A deficiency in juvenile and adult ADPKD mouse models.

\section{Results}

Perinatal deletion of $T h m 1$ in $P k d 2$ conditional knock-out mice reduces cortical collecting duct cystogenesis, but does not improve kidney function

To examine the effect of IFT-A deficiency in a rapidly progressing ADPKD mouse model, we deleted Thm1 together with $P k d 2$ at postnatal day (P) 0, and examined the renal phenotypes of control, Thm 1 conditional knock-out (cko), $P k d 2$ cko and $P k d 2$; Thm1 double knock-out (dko) mice at P21. At this early stage, Thm1 cko mice on a pure C57BL6/J background showed kidneys with some cortical 
tubular dilations, reduced kidney weight/body weight (KW/BW) ratios, and elevated blood urea nitrogen (BUN) levels (Figures 1A-1C). Pkd2 cko mice showed cysts in both renal cortex and medulla, as well as increased KW/BW ratios and BUN levels. Relative to $P k d 2$ cko mice, $P k d 2$; Thm 1 dko mice showed decreased cystogenesis specifically in the cortex (Figures 1D-1F), reduced KW/BW ratios, but similar BUN levels, indicating kidney dysfunction was not improved.

We examined the tubular origin of the renal cortical dilations and cysts. Thm 1 cko renal cortical dilations were mostly LTL+, indicating proximal tubules, while fewer were THP + or DBA+, marking loop of Henle and collecting duct, respectively (Figure 1G). Pkd2 cko renal cortices showed LTL+ dilations, THP+ cysts, and multiple, large DBA+ cysts. Relative to Thm 1 cko and Pkd2 cko kidneys, Pkd2; Thm1 dko cortices showed greater LTL+ dilations (Figures 1G, 1H). Relative to Pkd2 cko kidneys, Pkd2; Thm1 dko cortices showed similar THP+ cystogenesis (Figures 1G, S1A), but decreased DBA+ cysts (Figures 1G, 1I). Glomerular dilations were also present in Pkd2 cko kidneys, and increased in Pkd2;Thm1 dko kidneys (Figures 1J, 1K). These data reveal nephron segment-specific effects of Thm1 deletion on a $P k d 2$ cko background.

\section{$P k d 2$ deletion increases cilia length on renal epithelia}

In control kidneys, ciliary axonemes marked by acetylated $\alpha$-tubulin were $3.0 \mu \mathrm{m}$ and $2.1 \mu \mathrm{m}$ for LTL + and DBA+ cortical tubular epithelial cells, respectively (Figure 2). We also noted qualitative differences between LTL+ and DBA+ primary cilia, with the former cilia being thinner and longer, and the latter being thicker and more rod-like. Cilia lengths of LTL+ and DBA+ cells were increased in $P k d 2$ cko cortices. However, relative to $P k d 2$ cko cells, cilia lengths of $P k d 2 ; T h m 1$ dko LTL + cells were further increased, but similar for DBA+ cells. These differences reveal tubular-specific effects on cilia phenotype. 
ARL13B is a ciliary membrane protein essential for ciliogenesis ${ }^{27}$, and in cultured Thm1-null mouse embryonic fibroblasts, ARL13B in cilia is reduced ${ }^{23}$. We therefore examined whether ARL13B may have a role in the renal ciliary phenotypes. However, in DBA+ cortical renal epithelial cells, ARL13B intensities were similar across the mutant genotypes (Figure S2).

We examined whether Thml expression differed in proximal tubules versus cortical collecting duct. However, X-gal staining on kidneys of mice harboring a Thm1-lacZ allele showed relatively ubiquitous expression in the cortex and present in both LTL+ and cortical DBA+ tubules (Figure S3).

\section{Thm1 deletion increases inflammation}

To further evaluate the effect of Thml deletion on disease severity, we examined proliferation. Nuclear staining of proliferating cell nuclear antigen (PCNA) of LTL + tubules was similar across control, Thm1 cko and $P k d 2$ cko, but slightly elevated in $P k d 2$; Thm 1 dko kidneys (Figures 3A, 3B). In contrast, PCNA+ nuclei were increased in $P k d 2$ cko and $P k d 2$; Thm1 dko DBA+ non-cystic tubules, and further increased in $P k d 2$ cko and $P k d 2$; Thm 1 dko DBA+ cysts (Figure 3C). These data support that increased proliferation is an early driver of ADPKD renal cystogenesis.

Cyst growth compresses surrounding parenchyma, leading to injury, inflammation, and fibrosis. We immunostained kidney sections for alpha smooth muscle actin ( $\alpha$ SMA) and F4/80 to examine the presence of myofibroblasts and macrophages, respectively, which contribute to pro-inflammatory and pro-fibrotic processes. While control kidneys showed $\alpha$ SMA around blood vessels and a few F4/80+ cells, Thm 1 cko kidneys showed increased $\alpha$ SMA around glomeruli and tubular dilations, and $P k d 2$ cko and $P k d 2 ; T h m 1$ dko kidney showed even greater $\alpha \mathrm{SMA}+$ and increased F4/80+ labelling surrounding glomeruli, tubular dilations and cysts (Figure 3D-3F). Additionally, transcripts of inflammatory molecules, $\operatorname{Tn} f \alpha$ and $\operatorname{Tg} f \beta$, were increased in $\operatorname{Thm} 1$ cko renal extracts, while those of $I l 6, \operatorname{Tn} f \alpha, \operatorname{Tg} f \beta$, Ccl2 and Glil were increased in $P k d 2$ cko and $P k d 2 ; T h m 1$ dko kidney extracts (Figures 3G-3K). Thus, 
deletion of Thm1 alone increases inflammatory processes, and its deletion on a $P k d 2$ cko background results in similar or slightly increased ADPKD inflammation at P21.

\section{Perinatal deletion of Thm1 increases ERK and STAT3 signaling}

Increased ERK and STAT3 signaling promote disease progression in ADPKD ${ }^{28-31}$. ERK activation promotes cell proliferation ${ }^{30,32}$ and also acts upstream of mTOR and AMPK pathways regulating cellular metabolism ${ }^{33}$. STAT3 activation can have proliferative and inflammatory roles ${ }^{31,34,}$ 35. At P21, P-ERK did not localize with LTL, but localized with DBA (Figure 4A). While a similar number of P-ERK+ tubules was observed across control and the mutant genotypes (Figure 4B), P-ERK intensity was increased in dilated tubules of Thm 1 cko mice, and further increased in cyst-lining cells of $P k d 2$ cko and $P k d 2 ; T h m 1$ dko mice (Figure 4C). These data support that ERK activation is a driver of renal cyst growth. Using immunohistochemistry, P-STAT3 was revealed to be increased in epithelial cells lining cortical dilations in $T h m 1$ cko kidneys and to be increased further in cyst-lining cells of $P k d 2$ cko and $P k d 2$; Thm 1 dko kidneys, both in the cortex and medulla (Figure 4E). Western blot analyses also reveal increased ERK and STAT signaling in $P k d 2$ cko and $P k d 2 ; T h m 1$ dko renal extracts (Figures $4 \mathrm{~F}$, 4G). Thus, Thm1 loss as well as $P k d 2$ cystic disease increase ERK and STAT3 signaling.

\section{O-GIcNAc is increased in dilated tubules and cysts}

Altered cellular metabolism has emerged as another component of ADPKD pathobiology $33,36-38$. One of these alterations includes the Warburg effect ${ }^{33}$, whereby cells preferentially convert the product of glycolysis, pyruvate, into lactate, even in the presence of oxygen, at the expense of pyruvate entering mitochondria to enable oxidative phosphorylation. The nutrient sensor, O-linked $\beta$-N-acetylglucosamine (O-GlcNAc) regulates the balance between glycolysis and oxidative phosphorylation ${ }^{39}$, as well as ciliary homeostasis ${ }^{40,41}$, both of which are altered in $\mathrm{ADPKD}^{33,42}$. We therefore hypothesized that O-GlcNAc 
bioRxiv preprint doi: https://doi.org/10.1101/2020.04.26.061796; this version posted July 13, 2021. The copyright holder for this preprint (which

was not certified by peer review) is the author/funder, who has granted bioRxiv a license to display the preprint in perpetuity. It is made available under ACC-BY-NC-ND 4.0 International license.

signaling is misregulated in PKD. Relative to control, Thm1 cko kidneys showed increased O-GlcNAc expression in nuclei of cells lining dilations in the cortex, while $P k d 2$ cko and $P k d 2$; Thm 1 dko kidneys showed increased OGlcNAc staining in cyst-lining cells in both the cortex and medulla (Figures 5A, S4). O-GlcNAC levels are regulated by two enzymes, O-GlcNAc transferase (OGT) and O-GlcNAcase (OGA), which transfers and removes the O-GlcNAc moiety on protein substrates, respectively. In Thm1 cko kidneys, OGT was increased in the cytoplasm and nuclei of cells lining cortical dilations. In $P k d 2$ cko and in Pkd2; Thm 1 dko mice, intense OGT staining was present in cyst-lining cells in the cortex and medulla. In control and Thm 1 cko kidneys, light OGA staining was present in cytoplasm of cortical and medullary tubules. In $P k d 2$ cko and in $P k d 2$; Thm 1 dko mice, OGA was increased in the nuclei and cytoplasm of cyst-lining cells in both the cortex and medulla. Western blot analyses showed increased O-GlcNAc levels in mutant renal extracts, with highest levels in $P k d 2$ cko renal extracts (Figures 5B, 5C). These data suggest that increased O-GlcNAc signaling is a feature of ADPKD.

\section{Deletion of $T h m 1$ in adult $P k d 2$ or $P k d 1$ conditional knock-out mice markedly attenuates ADPKD renal cystogenesis}

We next examined the role of IFT-A deficiency in slowly progressive adult ADPKD mouse models. We deleted Thm1 together with $P k d 1$ at P35 and examined renal phenotypes at 6 months of age. Thm1 cko mice showed normal kidney morphology and BUN levels (Figure S5A). In Pkd1 cko mice, renal cysts were mostly in the cortex, with the largest and most abundant cysts being DBA + , fewer cysts being THP+ and dilations being LTL+ (Figure 6A). Notably, all these features were reduced in Pkd1;Thm1 dko kidneys. Similarly, KW/BW ratios and BUN levels were elevated in Pkdl cko mice, and corrected in Pkd1; Thm1 dko mice (Figures 6B, 6C).

Since PKD2 mutations result in less severe PKD, we deleted Thm1 together with Pkd2 at P28 and examined renal phenotypes at 6 months of age. Thm 1 cko kidney morphology and BUN levels resembled control (Figure S5B). $P k d 2$ cko mice show renal cysts mostly in the cortex, with the largest 
cysts being DBA+, and smaller cysts being LTL+ or THP+ (Figure 6D). In contrast, in Pkd2;Thm1 dko mice, the $P k d 2$ cko cystic phenotype is largely corrected morphologically. In $P k d 2$ cko mice, KW/BW ratios were similar to control and BUN levels showed a trend toward a slight elevation, reflecting the mild disease induced in adulthood. In $P k d 2 ; T h m 1$ dko mice, $\mathrm{KW} / \mathrm{BW}$ ratios were slightly reduced, due to increased body weight caused by global deletion of $T h m 1^{25}$, and BUN levels showed a slightly decreasing pattern relative to $P k d 2$ cko mice.

\section{Deletion of Thm1 in adult ADPKD mouse models reduces cilia lengths of cortical collecting duct} renal epithelia

Similar to juvenile ADPKD models, cilia lengths of $P k d 1$ cko and $P k d 2$ cko DBA+ adult renal epithelial cells were increased (Figures 6G, 6H, 6J, 6K). However, in contrast to juvenile models, cilia lengths of $P k d 1$; Thm 1 and $P k d 2$; Thm 1 dko DBA+ epithelia were reduced relative to those of $P k d 1$ cko and $P k d 2$ cko epithelia and similar to control. Additionally, human ADPKD sections had longer renal epithelial cilia than normal human kidney (NHK) sections (Figures 6I, 6L), supporting that increased cilia length is also a feature of the human disease ${ }^{22}$.

\section{Deletion of $\mathbf{T h m 1}$ in adult $P k d 1$ conditional knock-out mice reduces proliferation, inflammation,}

\section{P-ERK, P-STAT3, and O-GIcNAc}

We examined the extent of ADPKD attenuation by Thml deletion. Pkdl cko kidneys showed increased PCNA in cyst-lining cells, as well as increased $\alpha$ SMA and F4/80 around cysts and glomeruli, which were markedly reduced in $P k d 1$; Thm l dko kidneys (Figures 7A-D). Transcripts of proinflammatory molecules, $C c l 2, I l 6, \operatorname{Tnf} \alpha, \operatorname{Tg} f \beta, C c l 2$ and Gli1, were elevated in Pkdl cko kidneys, but reduced in Pkdl;Thml dko kidneys (Figure 7E). Similarly, P-ERK and P-STAT3 immunostaining was 
increased in Pkdl cko kidneys and reduced in Pkd1;Thml dko kidneys (Figures 7J, 7K). Thus, proliferative and pro-inflammatory pathways are attenuated in late-onset ADPKD by deletion of $T h m 1$.

Finally, we examined O-GlcNAcylation. In Pkdl cko kidneys, increased O-GlcNAc was present in cyst-lining epithelia of the cortex and in proteinaceous substances within some cysts, and in tubules of the medulla (Figures 8A, S6). OGT and OGA were also increased in cyst-lining cells of the cortex and in some tubules of the medulla. In Pkd1;Thm1 dko kidneys, O-GlcNAc, OGT and OGA staining were reduced. Western blot showed increased O-GlcNAc levels in $P k d l$ cko renal extracts (Figure 8B), and levels were reduced in $P k d 1$; Thm 1 dko extracts. These data suggest that increased O-GlcNAcylation is a feature also of slowly progressive ADPKD mouse models. Further, deletion of Thm1 in adult ADPKD mouse models attenuates perturbation of this metabolic regulator.

\section{Discussion}

To expand on the ciliary components whose dysfunction in ADPKD mouse models can attenuate disease severity $^{19,21,43}$, we examined the role of deletion of IFT-A gene, Thm1. Distinct from IFT-B, deletion of Thml does not improve kidney function in a juvenile ADPKD mouse model, but lessens most disease aspects in an adult model (Figure 9). Distinct from IFT-A adaptor, Tulp3, deletion of Thm1 in a juvenile ADPKD mouse model had partially ameliorative effects, reducing KW/BW ratios and cortical collecting duct cystogenesis. Our data also reveal that the effects of Thm1 deletion in juvenile ADPKD mice are nephron-specific, with no effect on loop of Henle-derived cysts, and exacerbation of proximal tubular and glomerular dilations. This suggests varying microenvironments between nephron segments modify cilia dysfunction. The differential effects of Tulp3 or Thm1 deletion in juvenile versus adult ADPKD mouse models further suggest distinct microenvironments between developing versus mature kidneys that influence not just renal cystogenesis, but also inflammation. 
Embryonic or perinatal mutation of Kif3a, Ift88, Tulp3 or Thm1 causes renal cystic disease 24,21 , 44, 45 , indicating that all these genes are required for kidney development. Thus, their differential effects in juvenile ADPKD mouse models may reflect differences in their cellular functions, such as cilia length regulation/ciliary homeostasis, ciliary entry or exit of different protein cargo, and regulation of signaling pathways. While IFT-B and -A complexes co-operate in ciliary homeostasis, these complexes also have distinct roles. IFT-B genes mediate anterograde IFT, while IFT-A genes mediate retrograde IFT as well as ciliary entry of membrane and signaling proteins. In contrast, Tulp3 does not mediate IFT, but is required for ciliary entry of certain membrane-associated and signaling molecules ${ }^{43}$. Additionally, IFTB and -A mutants can have opposing signaling phenotypes. Any of these functions can be explored to account for the differing juvenile dko phenotypes.

Increased cilia lengths on renal epithelia of several ADPKD mouse models, $P K D 1^{R C / R C}$, $P k d 1$ and $P k d 2$ cko mice ${ }^{46,47}$, and recently, on human ADPKD tissue ${ }^{22}$, have been reported. Our data substantiate that polycystin dysfunction causes increased cilia length in mouse models as well as in human ADPKD, suggesting ciliary mechanisms are likely conserved between mouse and human. Further, our data show that in addition to genotype, cilia structure varies by renal tubule segment and maturation, suggesting that factors within a tubule's microenvironment affect cilia length. Indeed, multiple factors including intracellular $\mathrm{Ca}^{2+}$ and cAMP, oxidative stress, cytokines, and fluid flow, affect cilia length of renal epithelial cells ${ }^{48-50}$, indicating that cilia length may be finely regulated in order to maintain renal tubular structure and function. In support of an ameliorative effect of reduced cilia length in ADPKD, inhibition of cilia disassembly in $P k d l$ cko mice increased renal cilia length and exacerbated $\mathrm{ADPKD}^{51}$, while in the $j c k$ non-orthologous PKD mouse model, which also has increased renal cilia lengths, pharmacological shortening of primary cilia was associated with attenuated $\mathrm{PKD}^{52}$.

Reduced Ccl2 and Wnt signaling in $P k d ;$ Ift-B dko mice ${ }^{20,22}$, and reduced P53 enhancing cilia disassembly in $P k d ;$ Ift-B dko cells ${ }^{42}$ are potential mechanisms by which ablation or shortening of 
primary cilia attenuate ADPKD. In addition to detecting ligands, primary cilia also detect mechanical cues. Although mechanosensing by primary cilia and the polycystins has been controversial, recent studies have renewed interest in a potential mechanosensory role for the polycystins, particularly regarding tissue microenvironment stiffness $^{53}$, 54. If sensing of physical forces in the tissue microenvironment is essential to maintaining renal tubular function, then other mechanical cues that would change with cyst growth include shear stress and intraluminal pressure. Cilia length itself could then also be a possible contributing factor in PKD severity. Supporting a role for cilia length, a recent study has shown that primary cilia of proximal tubule epithelial cells transduce shear stress into metabolic pathways that culminate in oxidative phosphorylation ${ }^{55}$. Finally, by extrapolating findings of cilia studies from the cancer field ${ }^{56}$, cilia of not only renal tubular epithelial cells, but of interstitial cells might also affect signaling and disease severity.

Our data are the first to reveal increased O-GlcNAcylation in cyst-lining renal epithelial cells of both juvenile and adult ADPKD mouse models, suggesting increased O-GlcNAcylation may be a feature of ADPKD. Increased O-GlcNAcylation is a pathologic feature of diabetic nephropathy ${ }^{57,58}$, and in rodent models, has shown to promote various aspects of chronic kidney disease ${ }^{59,60}$ and also renal fibrosis $^{61}$. In contrast, in a mouse model of contrast-induced acute kidney disease, an acute increase in O-GlcNAcylation was protective ${ }^{62}$, emphasizing important differences between chronic and acute increases in O-GlcNAcylation. While acute changes are adaptive and necessary to maintain cellular health and metabolism, chronic changes are likely to contribute to pathology ${ }^{63}$. Chronic elevation of OGlcNAcylation in cancer cells promotes tumor growth, and cancer cells show the Warburg effect, suggesting similar cellular metabolic alterations between ADPKD and cancer. A recent study has demonstrated that O-GlcNAcylation of Phosphoglycerate kinase 1 (PGK1), which catalyzes the first ATP molecule in glycolysis, activates PGK1 to enhance lactate production and reduce mitochondrial 
oxidative phosphorylation, promoting the Warburg effect ${ }^{39}$. Perhaps a similar mechanism may occur in ADPKD cells.

OGT localizes to the pericentriolar region during the early phases of ciliogenesis ${ }^{56}$, and perturbation of O-GlcNAcylation affects cilia length ${ }^{40,41}$. Further, the ciliary structural defects caused by $O g t$ deletion suggest impaired centriole formation and $\mathrm{IFT}^{56}$. Chronic hyper-O-GlcNAcylation in diabetic tissues results in ciliary defects ${ }^{64}$, demonstrating a causative link between misregulation of $\mathrm{O}$ GlcNAcylation and defective ciliary homeostasis in a disease context. Since increased renal cilia lengths are associated with ADPKD renal cystogenesis ${ }^{46,47}$, the regulation of O-GlcNAcylation on ciliogenesis could be another mechanism by which altered O-GlcNAcylation can affect ADPKD. Elucidating the mechanisms by which O-GlcNAc is upregulated in ADPKD and alters cellular metabolism and ciliogenesis could reveal novel mechanisms and therapeutic targets.

In summary, our data demonstrate for the first time the role of IFT-A deficiency in an ADPKD context, revealing differential effects between nephron segments and between developing and mature renal microenvironments. Our findings also reveal for the first time that O-GlcNAcylation is increased in ADPKD. We propose that as a regulator of ciliary homeostasis and of the balance between glycolysis and oxidative phosphorylation, increased O-GlcNAc may drive certain key ADPKD pathological processes.

\section{Materials and Methods}

\section{Generation of mice}

$P k d 1^{f l o x f l o x}, P k d 2^{f l o x f l o x}$ and ROSA26-Cre mice were obtained from the Jackson Laboratories (Stock numbers 010671, 017292 and 004847, respectively). Generation of Thm1 cko mice has been described previously ${ }^{24}:$ Thm $1^{\text {aln } /+} ;$ ROSA26Cre $e^{E R T+}$ male mice were mated to Thm $1^{\text {floxflox }}$ females. Pkd1 floxed alleles were introduced into the colony to generate Thm $1^{\text {flox/flox }}$; Pkd $1^{\text {flox/flox }}$ or 


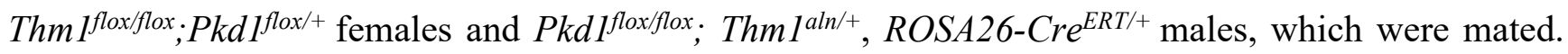
Similarly, $P k d 2$ floxed alleles were introduced into the colony to generate $T h m 1^{\text {floxflox }} ; P k d 2^{f l o x}$ flox or Thm 1 floxflox; $P k d 2^{f l o x /+}$ females and Pkd2floxflox; Thm $1^{a l n /+}, R O S A 26-C r e^{E R T /+}$ males. To generate early-

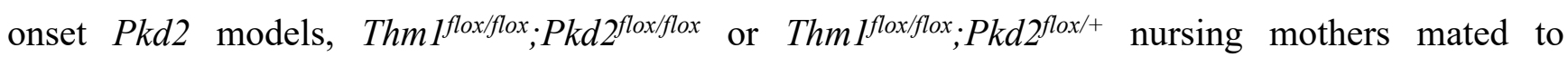
Pkd2floxflox; Thm $1^{a l n /+}$, ROSA26-Cre $e^{E R T /+}$ males were injected intraperitoneally with tamoxifen (8mg/40g; Sigma) at postnatal day 0 (P0) to induce gene deletion. Offspring were analyzed at P21. To generate late-onset $P k d 1$ models, offspring from matings between Thm $1^{\text {floxfllox; }}$ Pkd1 $1^{\text {floxflox }}$ or

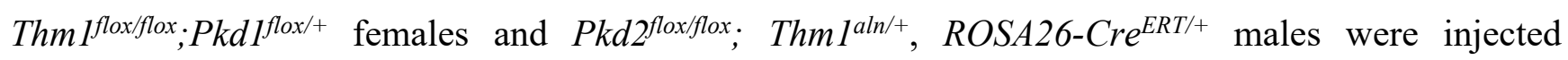
intraperitoneally with tamoxifen $(8 \mathrm{mg} / 40 \mathrm{~g})$ at P35. To generate late-onset $P k d 2$ models, offspring from

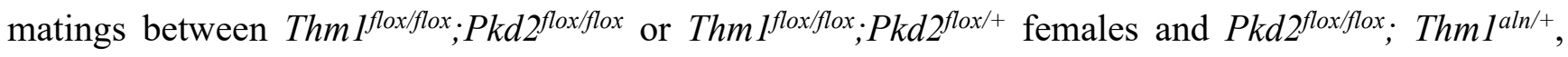
ROSA26-Cre $e^{E R T /+}$ males were injected intraperitoneally with tamoxifen $(8 \mathrm{mg} / 40 \mathrm{~g})$ at P28. Mice were analyzed at 6 months of age. All mouse lines were maintained on a pure C57BL6/J background (backcrossed 10 generations). All animal procedures were conducted in accordance with KUMCIACUC and AAALAC rules and regulations.

\section{Blood Urea Nitrogen Measurements}

Mouse trunk blood was collected in Microvette CB 300 Blood Collection System tubes (Kent Scientific), and centrifuged at $1800 \mathrm{~g}$ at room temperature for 10 minutes to collect serum. BUN was measured using the QuantiChrom Urea Assay Kit (BioAssay Systems) according to the manufacturer's protocol.

\section{Histology}

Kidneys were bisected transversely, fixed in $10 \%$ formalin for several days, then processed in a tissue processor and embedded in paraffin. Tissue sections $(7 \mu \mathrm{m})$ were obtained with a microtome. Sections were deparaffinized, rehydrated through a series of ethanol washes, stained with hematoxylin 
and eosin (H\&E), and mounted in Permount (ThermoFisher). Images were taken with a Nikon 80i microscope equipped with a Nikon DS-Fil camera. Cystic areas of H\&E-stained sections were quantified using ImageJ.

\section{Immunofluorescence}

Following deparaffinization and rehydration, tissue sections were subjected to antigen retrieval. Tissue sections were steamed for 15 minutes in Sodium Citrate Buffer (10 mM Sodium Citrate, $0.05 \%$ Tween 20, $\mathrm{pH} 6.0$ ), returned to room temperature, rinsed 10 times in distilled water, washed 5 minutes in PBS, incubated for 5 minutes in 1\% SDS in PBS based on a method by Brown et al., 1996 ${ }^{65}$, then washed 3 times in PBS. Sections were blocked with 1\% BSA in PBS for 1 hour at room temperature, then incubated with primary antibodies alone or together with lectins (Table S1) overnight at $4^{\circ} \mathrm{C}$. Sections were washed three times in PBS, then incubated with secondary antibodies (Table S2) for 1 hour. After three washes of PBS, sections were mounted with DAPI Fluoromount-G (Electron Microscopy Sciences). Staining was imaged using a Nikon 80i microscope with a photometrics camera or a Nikon Eclipse TiE attached to an A1R-SHR confocal, with an A1-DU4 detector, and LU4 laser launch.

\section{Immunohistochemistry}

Immunohistochemistry was performed as described ${ }^{66}$ using the following primary and secondary antibodies (Tables S1, S2). Once desired color was obtained, sections were counterstained with haemotoxylin. Staining was imaged using a Zeiss A1 microscope with a Axiocam 105 color camera.

\section{X-gal staining}

Kidneys of P0 mice harboring the Thm1-lacZ allele (KOMP Repository) were dissected, fixed for $5 \mathrm{~min}$ in $10 \%$ formalin, washed in lacZ wash buffer, then stained in $\mathrm{X}$-gal solution at $37^{\circ} \mathrm{C}$ overnight 
as described ${ }^{67}$. Kidneys were fixed in $10 \%$ formalin at $4^{0} \mathrm{C}$ overnight, processed, embedded in paraffin, and sectioned. Sections were rehydrated and either mounted in Permount or labelled with DBA or LTL lectin and mounted in DAPI Fluoromount-G. Sections were imaged using brightfield and confocal microscopy as described ${ }^{68}$.

\section{Western blot}

Kidney pieces (1/3 of kidney) were homogenized in Passive Lysis Buffer (Promega) containing protease inhibitors (Thermofisher). BCA assays (Pierce, ThermoFisher) were performed according to manufacturer's instructions. Western blots were performed as described ${ }^{66}$, using primary and secondary antibodies (Tables S1 and S2).

\section{Scanning electron microscopy}

SEM on renal tissue was performed as described ${ }^{69}$. Anesthetized mice were perfused with cold $2 \%$ glutaraldehyde in $0.1 \mathrm{M}$ cacodylate buffer, $\mathrm{pH}$ range $6.8-7.4$. Kidney cortices were dissected into small pieces and fixed in $2 \%$ glutaraldehyde in $0.1 \mathrm{M}$ cacodylate buffer at $4^{0} \mathrm{C}$ overnight, then washed in 0.1 M Na-cacodylate, $\mathrm{pH}$ 7.4. Samples were post-fixed with 1\% OsO4 in $0.1 \mathrm{M}$ Na-cacodylate buffer for 30 minutes, then dehydrated in an ethanol series, followed by hexamethyldisilazane (HMDS; Electron Microscopy Sciences). Samples were mounted onto metal stubs and sputter coated with gold. Samples were viewed and imaged using a Hitachi S-2700 Scanning Electron Microscope equipped with a Quartz PCI digital capture.

$q P C R$

RNA was extracted using Trizol (Life Technologies), then reverse transcribed into cDNA using Quanta Biosciences qScript cDNA mix (VWR International). qPCR was performed in duplicate using 
Quanta Biosciences Perfecta qPCR Supermix (VWR International) in a BioRad CFX Connect RealTime PCR Detection System with the following primers (Table S3) ${ }^{24,70}$.

\section{ADPKD and normal human kidney sections}

ADPKD (K386, K408, K423) and normal human kidney (NHK; K357, K402, K419) sections were obtained from the PKD Biomarkers, Biomaterials, and Cellular Models Core in the Kansas PKD Center. The protocol for the use of discarded human tissues complied with federal regulations and was approved by the Institutional Review Board at $\mathrm{KUMC}^{66}$.

\section{Statistics}

GraphPad Prism 8 software was used to perform statistical analyses. For analyses of more than two groups, ANOVA or Kruskal-Wallis tests were used to determine statistical significance $(\mathrm{p}<0.05)$ of data with or without a normal distribution, respectively. For analysis of two groups, an unpaired t-test was used.

\section{Acknowledgements}

We thank members of the KUMC Department of Anatomy and Cell Biology and the Jared Grantham Kidney Institute for helpful discussions. We thank Jing Huang of the KUMC Histology Core, which is supported by NIH U54HD090216 and NIH P30GM122731. We also thank Pat St. John and Larysa Stroganova of the KUMC Electron Microscopy Research Laboratory, which is supported by NIH P20GM104936. This work was also supported by a K-INBRE Summer Student Award to JTC (KINBRE P20GM103418), the PKD Biomaterials and Biomarkers Repository Core in the Kansas PKD Research and Translational Core Center (NIH P30DK106912 to JPC), R01DK108433 to MS, and R01DK103033 to PVT. 
bioRxiv preprint doi: https://doi.org/10.1101/2020.04.26.061796; this version posted July 13, 2021. The copyright holder for this preprint (which

was not certified by peer review) is the author/funder, who has granted bioRxiv a license to display the preprint in perpetuity. It is made available under aCC-BY-NC-ND 4.0 International license.

\section{Competing interests}

The authors declare no conflict of interest.

\section{Contributions}

WW, LMS, MAK, HHW, TSP, BAA, DTJ, RD, JTC, AC, MTP, MS, DPW, and PVT performed experiments. WW, LMS, MAK, HHW, TSP, BAA, DTJ, RD, JTC, AC, MTP, MS, CS, DPW, JPC and PVT analyzed and interpreted data. WW, LMS, BAA, and PVT designed research. WW, LMS, and PVT wrote the manuscript. All authors revised and approved the final manuscript. 


\section{References}

1. Lanktree MB, Haghighi A, Guiard E, et al. Prevalence Estimates of Polycystic Kidney and Liver Disease by Population Sequencing. J Am Soc Nephrol 2018; 29: 2593-2600.

2. Torres VE, Chapman AB, Devuyst O, et al. Tolvaptan in patients with autosomal dominant polycystic kidney disease. N Engl J Med 2012; 367: 2407-2418.

3. Blair HA. Tolvaptan: A Review in Autosomal Dominant Polycystic Kidney Disease. Drugs 2019.

4. Torres VE, Harris PC. Mechanisms of Disease: autosomal dominant and recessive polycystic kidney diseases. Nat Clin Pract Nephrol 2006; 2: 40-55; quiz 55.

5. Freedman BS, Lam AQ, Sundsbak JL, et al. Reduced ciliary polycystin-2 in induced pluripotent stem cells from polycystic kidney disease patients with PKD1 mutations. $J$ Am Soc Nephrol 2013; 24: 1571-1586.

6. Cai Y, Fedeles SV, Dong K, et al. Altered trafficking and stability of polycystins underlie polycystic kidney disease. J Clin Invest 2014; 124: 5129-5144.

7. Walker RV, Keynton JL, Grimes DT, et al. Ciliary exclusion of Polycystin-2 promotes kidney cystogenesis in an autosomal dominant polycystic kidney disease model. Nature communications 2019; 10: 4072. 
8. Fu W, Wang L, Kim S, et al. Role for the IFT-A Complex in Selective Transport to the Primary Cilium. Cell reports 2016; 17: 1505-1517.

9. Mukhopadhyay S, Wen X, Chih B, et al. TULP3 bridges the IFT-A complex and membrane phosphoinositides to promote trafficking of $\mathrm{G}$ protein-coupled receptors into primary cilia. Genes Dev 2010; 24: 2180-2193.

10. Liem KF, Jr., Ashe A, He M, et al. The IFT-A complex regulates Shh signaling through cilia structure and membrane protein trafficking. J Cell Biol 2012; 197: 789-800.

11. Yoder BK, Tousson A, Millican L, et al. Polaris, a protein disrupted in orpk mutant mice, is required for assembly of renal cilium. American journal of physiology Renal physiology 2002; 282: F541-552.

12. Jonassen JA, SanAgustin J, Baker SP, et al. Disruption of IFT complex A causes cystic kidneys without mitotic spindle misorientation. J Am Soc Nephrol 2012; 23: 641-651.

13. Lin F, Hiesberger T, Cordes K, et al. Kidney-specific inactivation of the KIF3A subunit of kinesin-II inhibits renal ciliogenesis and produces polycystic kidney disease. Proc Natl Acad Sci U S A 2003; 100: 5286-5291.

14. Davis EE, Zhang Q, Liu Q, et al. TTC21B contributes both causal and modifying alleles across the ciliopathy spectrum. Nat Genet 2011; 43: 189-196. 
15. Srivastava S, Molinari E, Raman S, et al. Many Genes-One Disease? Genetics of Nephronophthisis (NPHP) and NPHP-Associated Disorders. Front Pediatr 2017; 5: 287.

16. Tran PV, Haycraft CJ, Besschetnova TY, et al. THM1 negatively modulates mouse sonic hedgehog signal transduction and affects retrograde intraflagellar transport in cilia. Nat Genet 2008; 40: 403-410.

17. Qin J, Lin Y, Norman RX, et al. Intraflagellar transport protein 122 antagonizes Sonic Hedgehog signaling and controls ciliary localization of pathway components. Proceedings of the National Academy of Sciences of the United States of America 2011; 108: 1456-1461.

18. Eggenschwiler JT, Anderson KV. Cilia and developmental signaling. Annual review of cell and developmental biology 2007; 23: 345-373.

19. Ma M, Tian X, Igarashi P, et al. Loss of cilia suppresses cyst growth in genetic models of autosomal dominant polycystic kidney disease. Nat Genet 2013; 45: 1004-1012.

20. Viau A, Bienaime F, Lukas K, et al. Cilia-localized LKB1 regulates chemokine signaling, macrophage recruitment, and tissue homeostasis in the kidney. EMBO J 2018; 37.

21. Legue E, Liem KF, Jr. Tulp3 Is a Ciliary Trafficking Gene that Regulates Polycystic Kidney Disease. Curr Biol 2019; 29: 803-812 e805. 
22. Shao L, El-Jouni W, Kong F, et al. Genetic reduction of cilium-length by targeting intraflagellar transport 88 protein impedes kidney and liver cysts formation in mouse models of autosomal polycystic kidney disease. Kidney Int 2020.

23. Wang W, Allard BA, Pottorf TS, et al. Genetic interaction of mammalian IFT-A paralogs regulates cilia disassembly, ciliary entry of membrane protein, Hedgehog signaling, and embryogenesis. FASEB J 2020.

24. Tran PV, Talbott GC, Turbe-Doan A, et al. Downregulating hedgehog signaling reduces renal cystogenic potential of mouse models. J Am Soc Nephrol 2014; 25: 2201-2212.

25. Jacobs DT, Silva LM, Allard BA, et al. Dysfunction of intraflagellar transport-A causes hyperphagia-induced obesity and metabolic syndrome. Dis Model Mech 2016; 9: 789-798.

26. Piontek K, Menezes LF, Garcia-Gonzalez MA, et al. A critical developmental switch defines the kinetics of kidney cyst formation after loss of Pkd1. Nat Med 2007; 13: 1490-1495.

27. Lu H, Toh MT, Narasimhan V, et al. A function for the Joubert syndrome protein Arl13b in ciliary membrane extension and ciliary length regulation. Dev Biol 2015; 397: 225-236.

28. Takakura A, Nelson EA, Haque N, et al. Pyrimethamine inhibits adult polycystic kidney disease by modulating STAT signaling pathways. Hum Mol Genet 2011; 20: 4143-4154. 
29. Yamaguchi T, Pelling JC, Ramaswamy NT, et al. cAMP stimulates the in vitro proliferation of renal cyst epithelial cells by activating the extracellular signal-regulated kinase pathway. Kidney Int 2000; 57: 1460-1471.

30. Yamaguchi T, Nagao S, Wallace DP, et al. Cyclic AMP activates B-Raf and ERK in cyst epithelial cells from autosomal-dominant polycystic kidneys. Kidney Int 2003; 63: 1983-1994.

31. Talbot JJ, Shillingford JM, Vasanth S, et al. Polycystin-1 regulates STAT activity by a dual mechanism. Proc Natl Acad Sci U S A 2011; 108: 7985-7990.

32. Yamaguchi T, Wallace DP, Magenheimer BS, et al. Calcium restriction allows cAMP activation of the B-Raf/ERK pathway, switching cells to a cAMP-dependent growth-stimulated phenotype. J Biol Chem 2004; 279: 40419-40430.

33. Rowe I, Chiaravalli M, Mannella V, et al. Defective glucose metabolism in polycystic kidney disease identifies a new therapeutic strategy. Nat Med 2013; 19: 488-493.

34. Weimbs T, Talbot JJ. STAT3 Signaling in Polycystic Kidney Disease. Drug Discov Today Dis Mech 2013; 10: e113-e118.

35. Talbot JJ, Song X, Wang X, et al. The cleaved cytoplasmic tail of polycystin-1 regulates Srcdependent STAT3 activation. J Am Soc Nephrol 2014; 25: 1737-1748.

36. Torres JA, Kruger SL, Broderick C, et al. Ketosis Ameliorates Renal Cyst Growth in Polycystic Kidney Disease. Cell metabolism 2019; 30: 1007-1023 e1005. 
37. Leonhard WN, Song X, Kanhai AA, et al. Salsalate, but not metformin or canagliflozin, slows kidney cyst growth in an adult-onset mouse model of polycystic kidney disease. EBioMedicine 2019; 47: 436-445.

38. Flowers EM, Sudderth J, Zacharias L, et al. Lkb1 deficiency confers glutamine dependency in polycystic kidney disease. Nature communications 2018; 9: 814.

39. Nie H, Ju H, Fan J, et al. O-GlcNAcylation of PGK1 coordinates glycolysis and TCA cycle to promote tumor growth. Nature communications 2020; 11: 36 .

40. Tian JL, Qin H. O-GlcNAcylation Regulates Primary Ciliary Length by Promoting Microtubule Disassembly. iScience 2019; 12: 379-391.

41. Yu F, Li T, Sui Y, et al. O-GlcNAc transferase regulates centriole behavior and intraflagellar transport to promote ciliogenesis. Protein Cell 2020.

42. Gerakopoulos V, Ngo P, Tsiokas L. Loss of polycystins suppresses deciliation via the activation of the centrosomal integrity pathway. Life Sci Alliance 2020; 3.

43. Hwang SH, Somatilaka BN, Badgandi H, et al. Tulp3 Regulates Renal Cystogenesis by Trafficking of Cystoproteins to Cilia. Curr Biol 2019; 29: 790-802 e795.

44. Patel V, Li L, Cobo-Stark P, et al. Acute kidney injury and aberrant planar cell polarity induce cyst formation in mice lacking renal cilia. Hum Mol Genet 2008; 17: 1578-1590. 
45. Moyer JH, Lee-Tischler MJ, Kwon HY, et al. Candidate gene associated with a mutation causing recessive polycystic kidney disease in mice. Science 1994; 264: 1329-1333.

46. Hopp K, Ward CJ, Hommerding CJ, et al. Functional polycystin-1 dosage governs autosomal dominant polycystic kidney disease severity. J Clin Invest 2012; 122: 4257-4273.

47. Liu X, Vien T, Duan J, et al. Polycystin-2 is an essential ion channel subunit in the primary cilium of the renal collecting duct epithelium. eLife 2018; 7.

48. Besschetnova TY, Kolpakova-Hart E, Guan Y, et al. Identification of signaling pathways regulating primary cilium length and flow-mediated adaptation. Curr Biol 2010; 20: 182-187.

49. Kim JI, Kim J, Jang HS, et al. Reduction of oxidative stress during recovery accelerates normalization of primary cilia length that is altered after ischemic injury in murine kidneys. $\mathrm{Am}$ J Physiol Renal Physiol 2013; 304: F1283-1294.

50. Upadhyay VS, Muntean BS, Kathem SH, et al. Roles of dopamine receptor on chemosensory and mechanosensory primary cilia in renal epithelial cells. Front Physiol 2014; 5: 72.

51. Nikonova AS, Plotnikova OV, Serzhanova V, et al. Nedd9 restrains renal cystogenesis in Pkd1-/- mice. Proc Natl Acad Sci U S A 2014; 111: 12859-12864. 
52. Husson H, Moreno S, Smith LA, et al. Reduction of ciliary length through pharmacologic or genetic inhibition of CDK5 attenuates polycystic kidney disease in a model of nephronophthisis. Hum Mol Genet 2016.

53. Cruz NM, Song X, Czerniecki SM, et al. Organoid cystogenesis reveals a critical role of microenvironment in human polycystic kidney disease. Nat Mater 2017; 16: 1112-1119.

54. Nigro EA, Distefano G, Chiaravalli M, et al. Polycystin-1 Regulates Actomyosin Contraction and the Cellular Response to Extracellular Stiffness. Scientific reports 2019; 9: 16640.

55. Miceli C, Roccio F, Penalva-Mousset L, et al. The primary cilium and lipophagy translate mechanical forces to direct metabolic adaptation of kidney epithelial cells. Nature cell biology 2020; 22: 1091-1102.

56. Kiseleva AA, Korobeynikov VA, Nikonova AS, et al. Unexpected Activities in Regulating Ciliation Contribute to Off-target Effects of Targeted Drugs. Clin Cancer Res 2019; 25: 41794193.

57. Degrell P, Cseh J, Mohas M, et al. Evidence of O-linked N-acetylglucosamine in diabetic nephropathy. Life Sci 2009; 84: 389-393.

58. Gellai R, Hodrea J, Lenart L, et al. Role of O-linked N-acetylglucosamine modification in diabetic nephropathy. American journal of physiology Renal physiology 2016; 311: F1172F1181. 
59. Silva-Aguiar RP, Bezerra NCF, Lucena MC, et al. O-GlcNAcylation reduces proximal tubule protein reabsorption and promotes proteinuria in spontaneously hypertensive rats. $J$ Biol Chem 2018; 293: 12749-12758.

60. Xu TH, Sheng Z, Li Y, et al. OGT knockdown counteracts high phosphate-induced vascular calcification in chronic kidney disease through autophagy activation by downregulating YAP. Life Sci 2020; 261: 118121.

61. Feng D, Sheng-Dong L, Tong W, et al. O-GlcNAcylation of RAF1 increases its stabilization and induces the renal fibrosis. Biochim Biophys Acta Mol Basis Dis 2020; 1866: 165556.

62. $\mathrm{Hu} \mathrm{J}$, Chen $\mathrm{R}$, Jia $\mathrm{P}$, et al. Augmented O-GlcNAc signaling via glucosamine attenuates oxidative stress and apoptosis following contrast-induced acute kidney injury in rats. Free Radic Biol Med 2017; 103: 121-132.

63. Ong Q, Han W, Yang X. O-GlcNAc as an Integrator of Signaling Pathways. Front Endocrinol (Lausanne) 2018; 9: 599.

64. Yu F, Guo S, Li T, et al. Ciliary defects caused by dysregulation of O-GlcNAc modification are associated with diabetic complications. Cell Res 2019; 29: 171-173.

65. Brown D, Lydon J, McLaughlin M, et al. Antigen retrieval in cryostat tissue sections and cultured cells by treatment with sodium dodecyl sulfate (SDS). Histochem Cell Biol 1996; 105: 261-267. 
66. Silva LM, Jacobs DT, Allard BA, et al. Inhibition of Hedgehog signaling suppresses proliferation and microcyst formation of human Autosomal Dominant Polycystic Kidney Disease cells. Scientific reports 2018; 8: 4985.

67. Stottmann RW, Tran PV, Turbe-Doan A, et al. Ttc21b is required to restrict sonic hedgehog activity in the developing mouse forebrain. Dev Biol 2009; 335: 166-178.

68. Levitsky KL, Toledo-Aral JJ, Lopez-Barneo J, et al. Direct confocal acquisition of fluorescence from X-gal staining on thick tissue sections. Scientific reports 2013; 3: 2937.

69. Silva LM, Wang W, Allard BA, et al. Analysis of primary cilia in renal tissue and cells. Methods Cell Biol 2019; 153: 205-229.

70. Dwivedi N, Tao S, Jamadar A, et al. Epithelial Vasopressin Type-2 Receptors Regulate Myofibroblasts by a YAP-CCN2-Dependent Mechanism in Polycystic Kidney Disease. J Am Soc Nephrol 2020; 31: 1697-1710. 


\section{Figure Legends}

Figure 1. Thm1 deletion in juvenile $P$ kd 2 conditional knock-out mice reduces cortical cystogenesis.

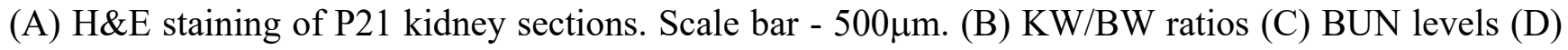
Percent cystic index of whole kidney; (E) of cortex; (F) and of medulla. Pkd2 cko values are set at 1. Bars represent mean \pm SD. Statistical significance was determined by Brown-Forsythe and Welch ANOVA tests followed by Dunnett's T3 multiple comparisons test in (B), and ordinary one-way ANOVA test followed by Sidak's multiple comparisons test in (C). ***p $<0.001, * * * * p<0.0001$, compared to Ctrl. In (B) and (C), unpaired t-test was used to determine the difference between $P k d 2$ and dko groups. ${ }^{\# \#} \mathrm{p}<0.05$. In (D), (E) and (F), statistical significance was determined by unpaired two-tailed t-test. $* * \mathrm{p}<0.01, * * * * \mathrm{p}<0.0001$, compared to Ctrl. Each data point represents a mouse. (G) Staining of kidney cortex with LTL, THP and DBA. Scale bar - $100 \mu \mathrm{m}(\mathrm{H})$ Quantification of LTL+ dilations and (I) DBA+ cystic index in renal cortex. $P k d 2$ cko values are set at 1 . Bars represent mean \pm SD. Statistical significance was determined by unpaired two-tailed t-test. ${ }^{*} \mathrm{p}<0.05,{ }^{*} * \mathrm{p}<0.001$, compared to Ctrl. (J)

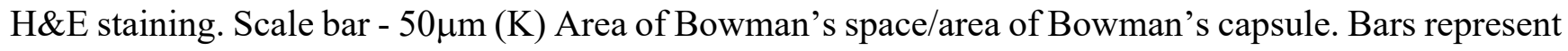
mean \pm SD. Statistical significance was determined by Kruskal-Wallis test followed by Dunn's multiple comparisons test. ${ }^{\# \# \#} \mathrm{p}<0.0001 ;{ }^{* *} \mathrm{p}<0.01$ compared to $\mathrm{Ctrl} ;{ }^{* * * * *} \mathrm{p}<0.0001$ compared to $\mathrm{Ctrl}$

Figure 2. Perinatal $P \boldsymbol{k d} 2$ deletion increases renal epithelial primary cilia lengths (A) Images of kidney cortex immunostained for acetylated $\alpha$-tubulin (red) together with LTL (green). Scale bar - $10 \mu \mathrm{m}$. (F) Quantification of cilia length of LTL+ cells. (G) Immunostaining of kidney cortex for acetylated $\alpha$ tubulin and IFT81 together with DBA. Scale bar - 10 $\mathrm{m}$. n=3 mice/genotype. Quantification of cilia length of cortical DBA+ cells. Bars represent mean \pm SD. Statistical significance was determined by Kruskal-Wallis test followed by Dunn's multiple comparisons test. ${ }^{*} \mathrm{p}<0.05, * * * * \mathrm{p}<0.0001$, compared 


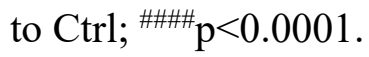

Figure 3. Perinatal Thm1 deletion increases inflammation. (A) Images of kidney cortex

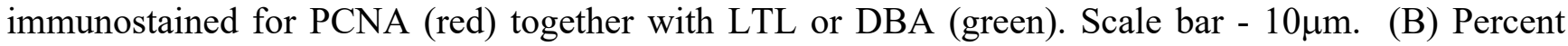
PCNA+ cells per LTL+ and (C) DBA+ tubule. Each dot represents a tubule or a cyst from $n=3$ mice/genotype. Bars represent mean \pm SD. Statistical significance was determined by Kruskal-Wallis test followed by Dunn's multiple comparisons test. ${ }^{*} \mathrm{p}<0.05, * * * \mathrm{p}<0.001, * * * * \mathrm{p}<0.0001$, compared to Ctrl. Unpaired t-test was used to determine the difference between non-cystic tubules and cysts. ${ }^{\#} \mathrm{P}<0.05$ compared to $P k d 2$ cko non-cystic tubule; ${ }^{\&} \mathrm{P}<0.05$ compared to dko non-cystic tubule. (D)

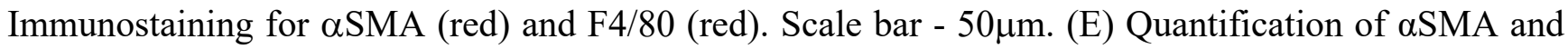
(F) F4/80 staining. Each dot represents percentage of staining of a 20X or 40X image (4-5 images/mouse) from $n=3$ mice/genotype. (G, H, I, J, K) qPCR. Each dot represents a mouse. In (E) and (G), statistical significance was determined by Brown-Forsythe and Welch ANOVA tests followed by Dunnett's T3 multiple comparisons test. In (F), (H), (J) and (K), statistical significance was determined by KruskalWallis test followed by Dunn's multiple comparisons test. In (I), statistical significance was determined by ordinary one-way ANOVA followed by Sidak's multiple comparisons test. ${ }^{*} \mathrm{p}<0.05,{ }^{*} \mathrm{p}<0.01$, $* * * \mathrm{p}<0.001, * * * * \mathrm{p}<0.0001$, compared to Ctrl. Bars represent mean $\pm \mathrm{SD}$.

Figure 4. Perinatal Thm 1 deletion increases ERK and STAT3 activation. (A) Images of kidney cortex immunostained for P-ERK together with LTL or DBA. Scale bar - 50 $\mu \mathrm{m}$. (B) Quantification of $\mathrm{P}-\mathrm{ERK}+$ tubules or cysts and (C) P+ERK intensity. Each dot represents a tubule or cyst from $\mathrm{n}=3$ mice/genotype. Statistical significance was determined by Kruskal-Wallis test followed by Dunn's multiple comparisons test. $* * * \mathrm{p}<0.001, * * * * \mathrm{p}<0.0001$, compared to Ctrl. (D) Immunohistochemistry for P-STAT3. $\mathrm{n}=3$ mice/genotype. Scale bar - 25 $\mu \mathrm{m}$. (E) Western blot for STAT3 and ERK signaling, 
(F) Quantification of P-STAT3/STAT3 and (G) of P-ERK/ERK ratios. Statistical significance was determined by ordinary one-way ANOVA test followed by Sidak's multiple comparisons test or BrownForsythe and Welch ANOVA tests followed by Dunnett's T3 multiple comparisons test, respectively. ${ }^{*} \mathrm{p}<0.05, * * * * \mathrm{p}<0.0001$, compared to $\mathrm{Ctrl} .{ }^{*} \mathrm{p}<0.05$.

Figure 5. O-GIcNAc is increased in cells lining dilations and cysts in juvenile mice. (A) Images of kidney cortex following immunohistochemistry for O-GlcNAc, OGT and OGA. Scale bar - 25 $\mu$ m. n=3 mice/genotype. (B) Western blot of O-GlcNAc and (C) quantification. Statistical significance was determined by ordinary one-way ANOVA test followed by Sidak's multiple comparisons test. * $\mathrm{p}<0.05$, $* * * \mathrm{p}<0.001$, compared to Ctrl. ${ }^{*} \mathrm{p}<0.05$.

Figure 6. Thm 1 deletion in adult ADPKD mouse models attenuates renal cystogenesis and cilia lengthening. (A) Histology, lectin staining and immunostaining for $P k d 1$ cko mice and (B) for $P k d 2$ cko mice. Scale bars - $100 \mu \mathrm{m}$ and $50 \mu \mathrm{m}$, respectively. (C) KW/BW ratios and (D) BUN levels for Pkdl cko mice and $(\mathrm{E}, \mathrm{F})$ for $P k d 2$ cko mice. Bars represent mean \pm SD. Each dot represents an animal. In (C), statistical significance was determined by Brown-Forsythe and Welch ANOVA tests followed by Dunnett's T3 multiple comparisons test. In (D) and (E), statistical significance was determined by oneway ANOVA followed by Tukey's test. In (F), statistical significance was determined by Kruskal-Wallis test followed by Dunn's multiple comparisons test. ${ }^{*} \mathrm{p}<0.05,{ }^{*} \mathrm{p}<0.01$, compared to Ctrl; ${ }^{\# \#} \mathrm{p}<0.01$; ${ }^{\# \# \#} \mathrm{p}<0.001$. (G) Images of $P k d 1$ cko kidney cortex immunostained for acetylated $\alpha$-tubulin together with DBA. Scale bar - $10 \mu \mathrm{m}$. Scanning electron micrographs of primary cilia. Scale bar $-1.5 \mu \mathrm{m} . \mathrm{n}=3$ mice/genotype. (H) Images of $P k d 2$ cko kidney cortex immunostained for acetylated $\alpha$-tubulin together with DBA. Scale bar - 10 $\mu \mathrm{m}$. n=3/genotype. (I) Immunostaining for ARL13B of normal human kidney (NHK) and ADPKD renal sections. Scale bar - 10 $\mu \mathrm{m} . \mathrm{n}=3$ for NHK and ADPKD. (J) Quantification of 
renal cilia lengths of $P k d 1$ cko mice, (K) $P k d 2$ cko mice, and (L) ADPKD tissue. Cilia lengths were quantified from immunofluorescence experiments of (G, H, I). Significance was determined using Kruskal-Wallis test followed by Dunn's multiple comparisons test (J, K) or by Mann Whitney test (L).

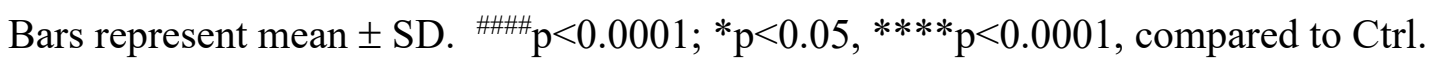

\section{Figure 7. Thm1 deletion in adult $P$ kdl conditional knock-out mice attenuates proliferation and} inflammation. (A) Images of kidney cortex immunostained for PCNA (red) together with DBA (green).

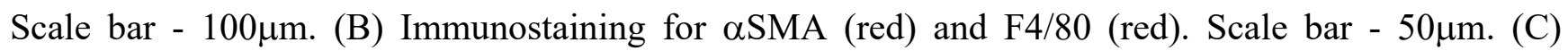
Quantification of $\alpha$ SMA and (D) F4/80 staining. Each dot represents percentage of staining of a $20 \mathrm{X}$ or 40X image ( 5 images/mouse) from $n=3$ mice/genotype. (E, F, G, H, I) qPCR. Each dot represents a mouse. In (C), (D), (E) and (H), statistical significance was determined by Kruskal-Wallis test followed by Dunn's multiple comparisons test. In (F) and (I), statistical significance was determined by ordinary one-way ANOVA test followed by Tukey's multiple comparisons test. In (G), statistical significance was determined by Brown-Forsythe and Welch ANOVA tests followed by Dunnett's T3 multiple comparisons test. ${ }^{*} \mathrm{p}<0.05,{ }^{* * *} \mathrm{p}<0.001$, compared to $\mathrm{Ctrl} ;{ }^{\#} \mathrm{p}<0.05,{ }^{\# \#} \mathrm{p}<0.01,{ }^{\# \#} \mathrm{p}<0.001$. Bars represent mean \pm SD. (J) Immunostaining for P-ERK (red) together with LTL (green) or DBA (green). Scale bar

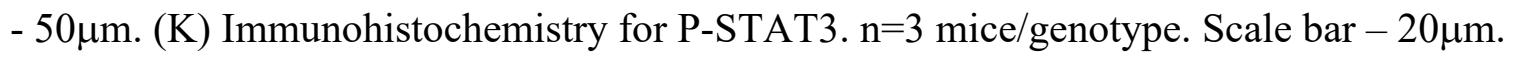

\section{Figure 8. Thm1 deletion in adult $P$ kd1 conditional knock-out mice attenuates increased O-GIcNAc.}

(A) Images of kidney cortex following immunohistochemistry for O-GlcNAc, OGT and OGA. Scale bar

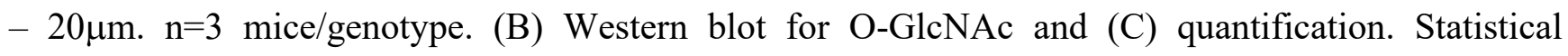
significance was determined by ordinary one-way ANOVA test followed by Tukey's multiple comparisons test. ${ }^{* *} \mathrm{p}<0.01$, compared to Ctrl. ${ }^{\# \#} \mathrm{p}<0.01$. 
bioRxiv preprint doi: https://doi.org/10.1101/2020.04.26.061796; this version posted July 13, 2021. The copyright holder for this preprint (which

was not certified by peer review) is the author/funder, who has granted bioRxiv a license to display the preprint in perpetuity. It is made available under aCC-BY-NC-ND 4.0 International license.

\section{Figure 9. Model of role of IFT-A deficiency in ADPKD.}

In an early-onset, juvenile ADPKD mouse model, deletion of Thm1 causes nephron segment-specific effects, attenuating cortical collecting duct (CD) cystogenesis, but worsening proximal tubular and glomerular dilations, without affecting kidney function, cilia length, inflammation, and ERK, STAT3, and O-GlcNAc signaling. In a late-onset, adult ADPKD mouse model, deletion of Thm1 improves kidney function and reduces cystogenesis, inflammation, cilia length, and ERK, STAT3, and O-GlcNAc signaling. 
A

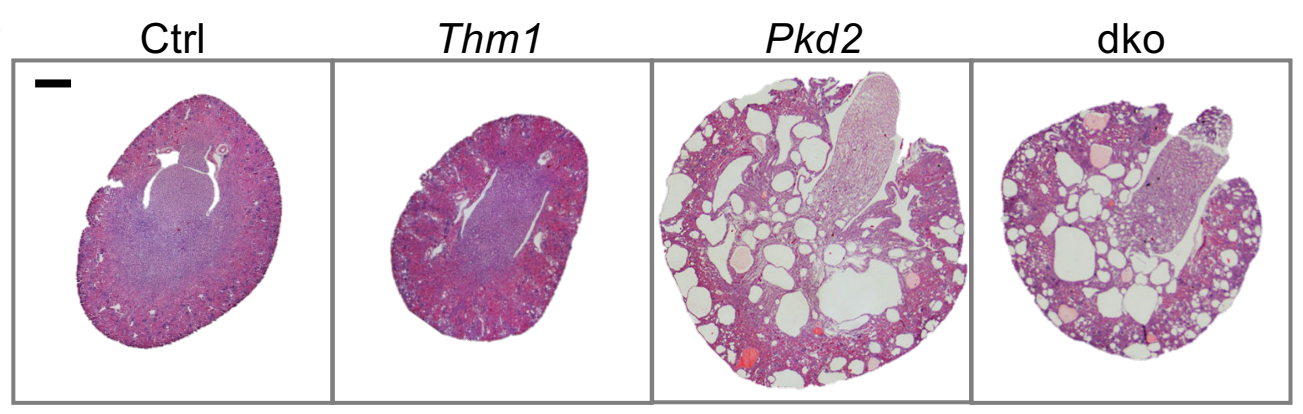

C

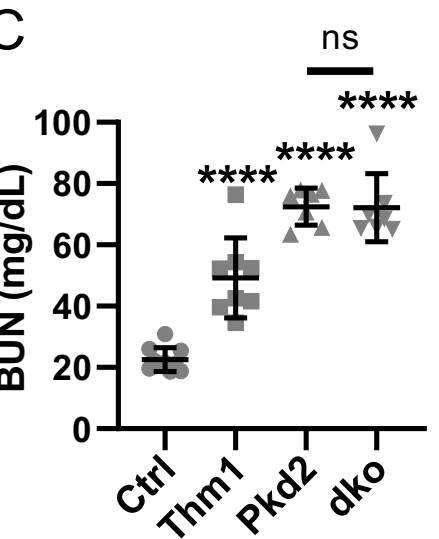

D

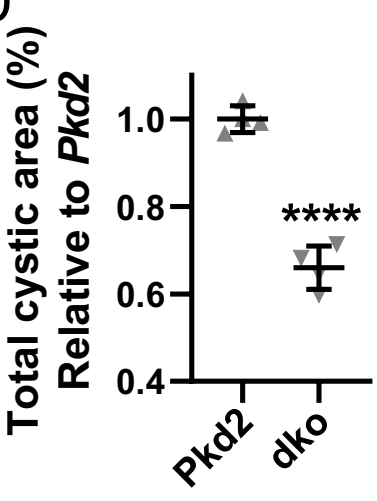

Eळ

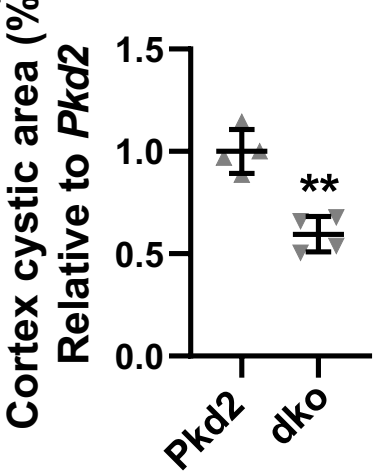

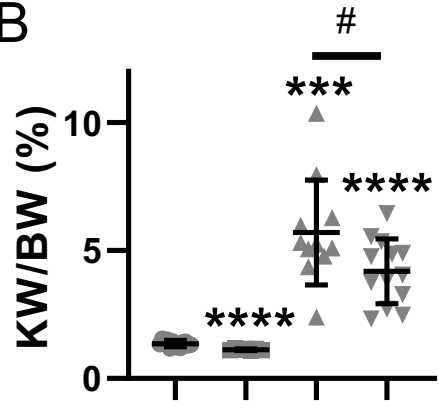

c"

$\mathrm{F} \stackrel{0}{\circ}$

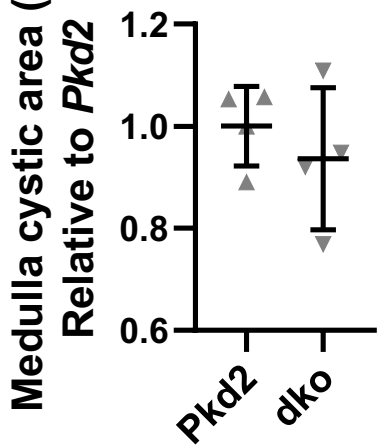

G

G Ctrl

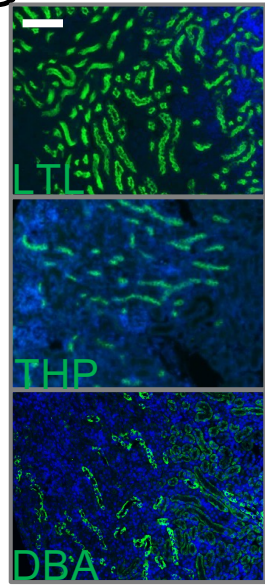

J

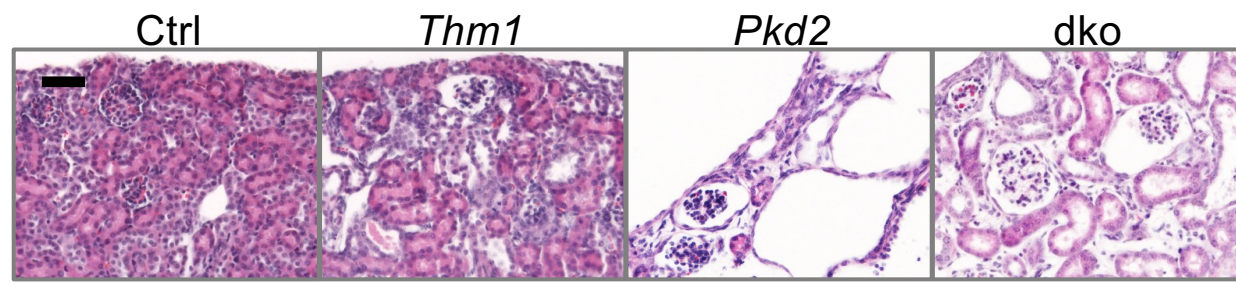

Pkd2

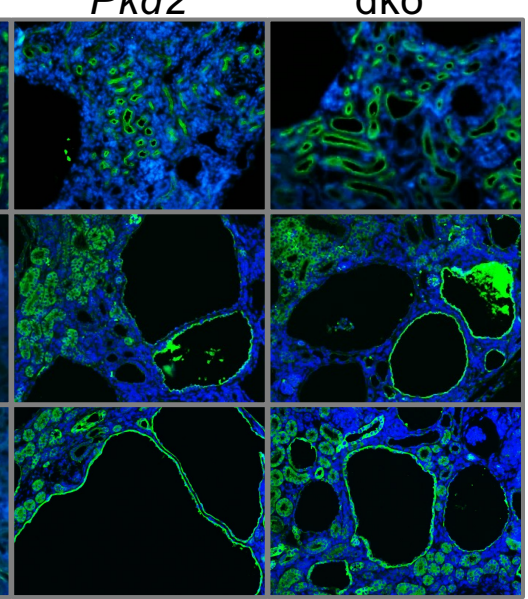

$\mathrm{H}$
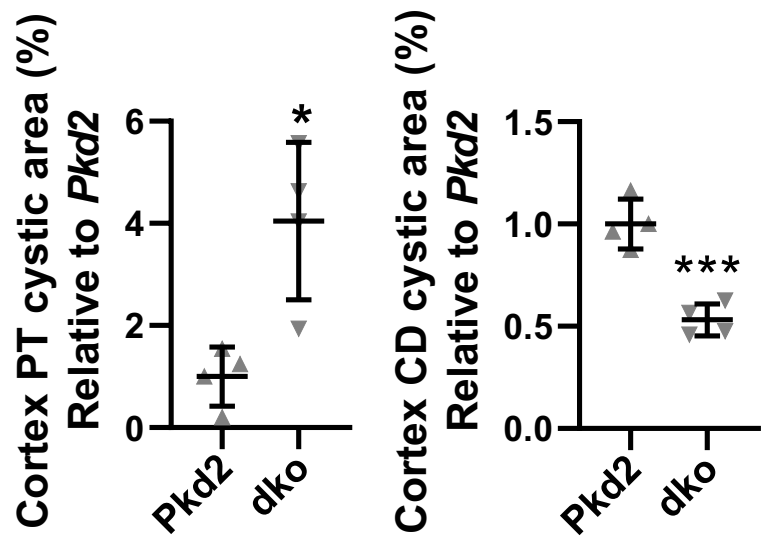

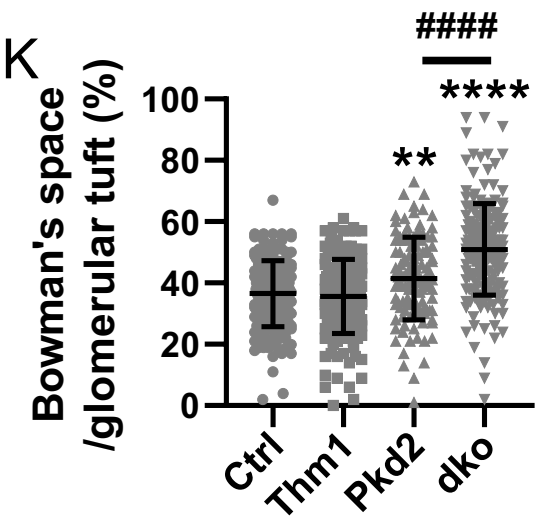




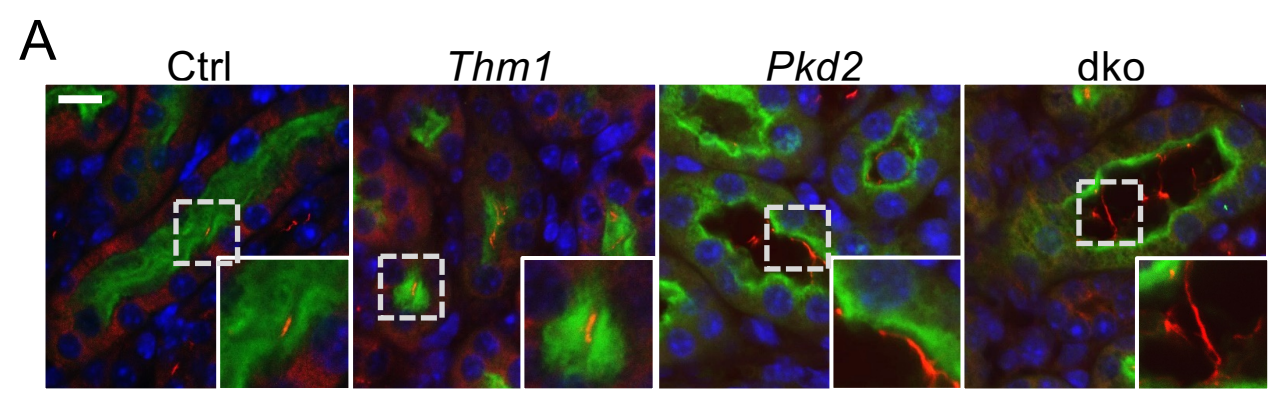

LTL/acet $\boldsymbol{\alpha}$-tub

C

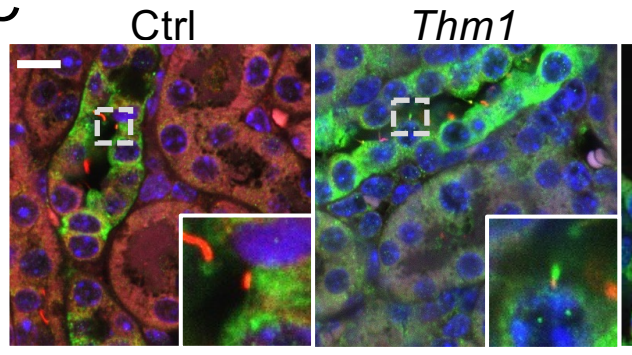

DBA/IFT81/acet $\boldsymbol{\alpha}$-tub

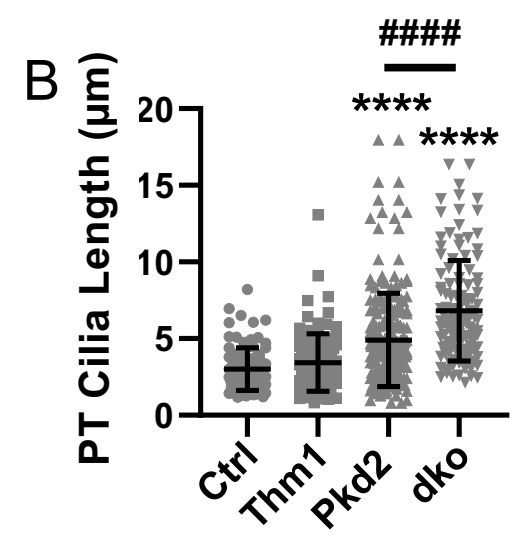

$P k d 2$

dko

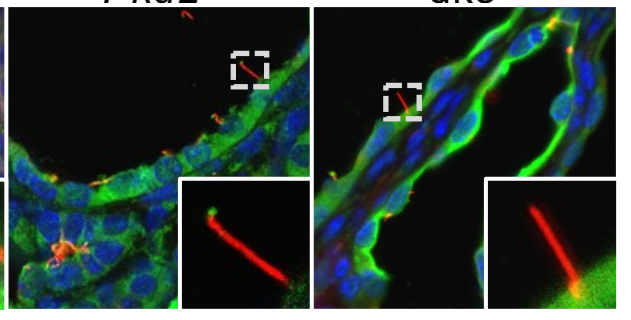

DE

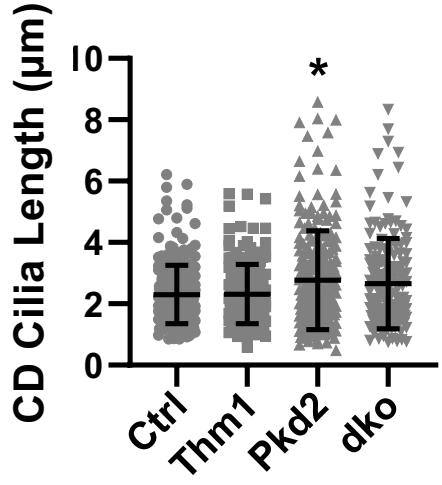


A

Ctrl

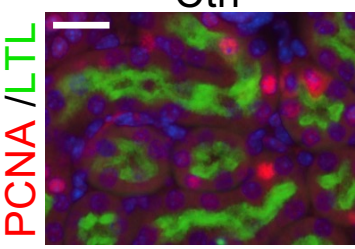

Thm1

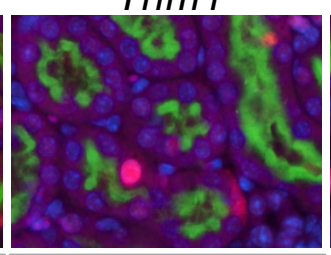

Pkd2
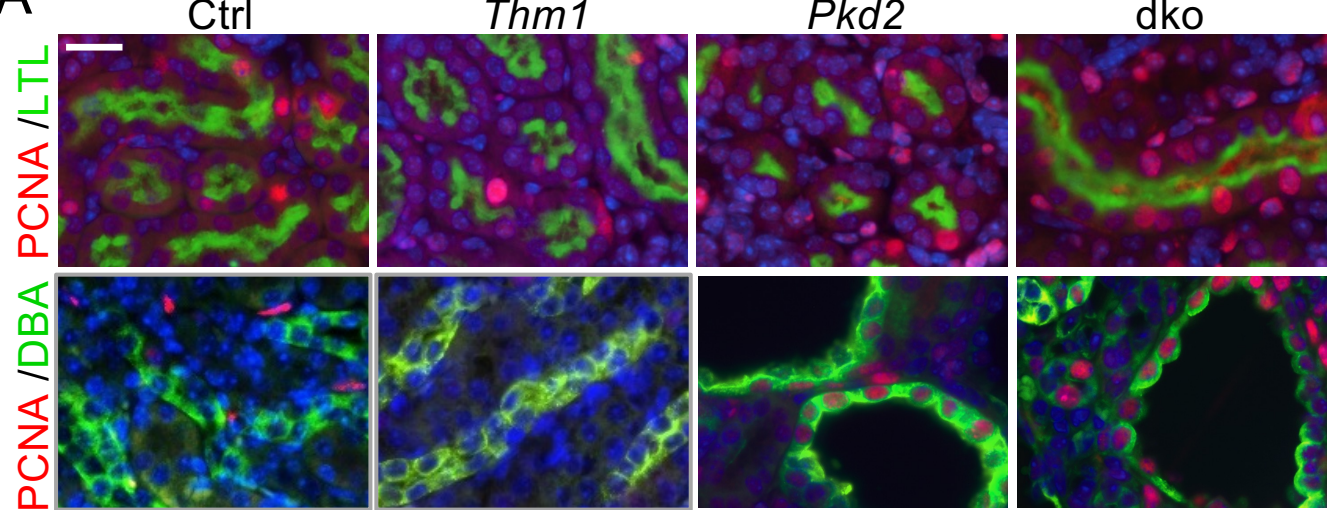

B

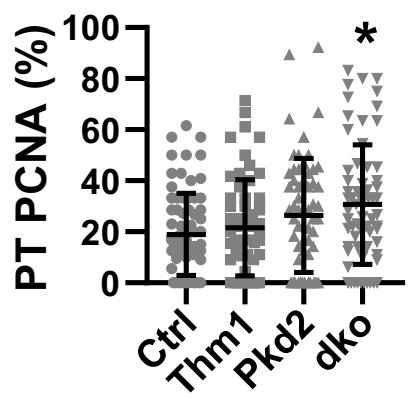

C

Tubule Cyst
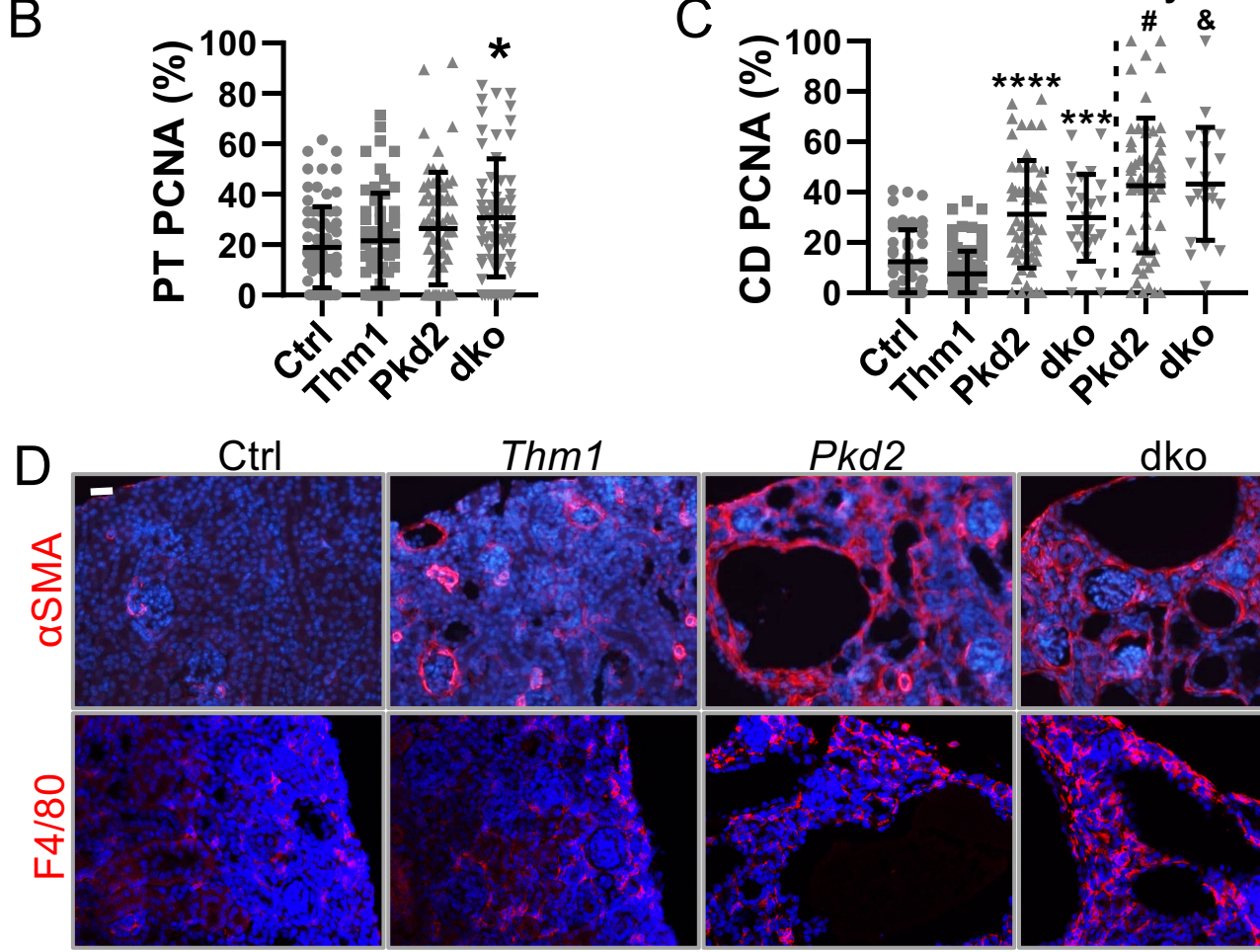

Thm1

Pkd2
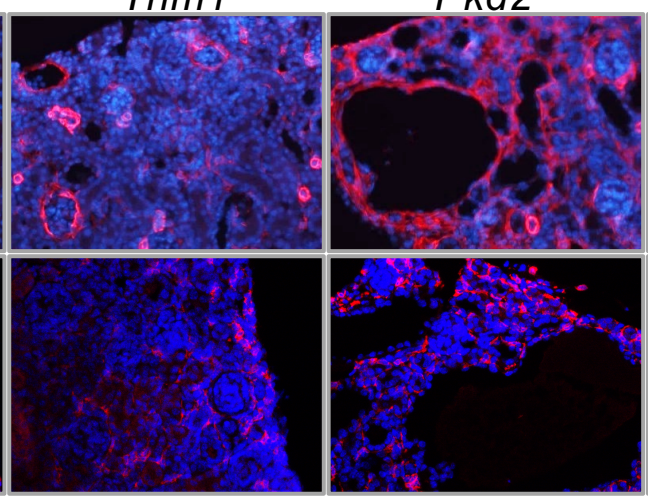

dko

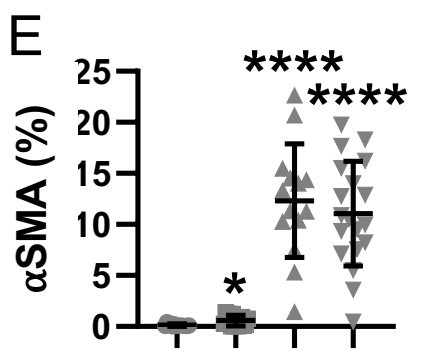

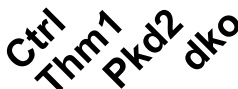

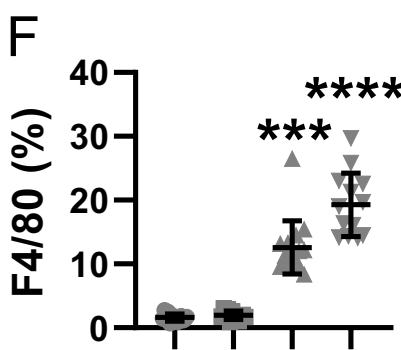

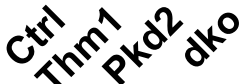

G

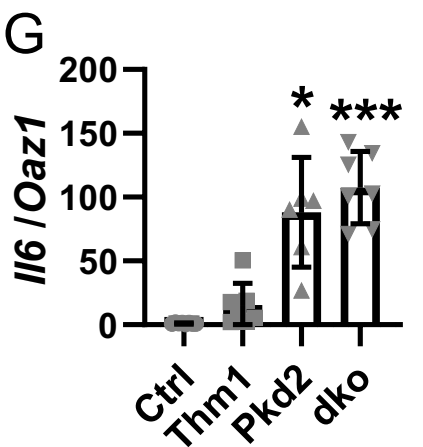

$\mathrm{H}$
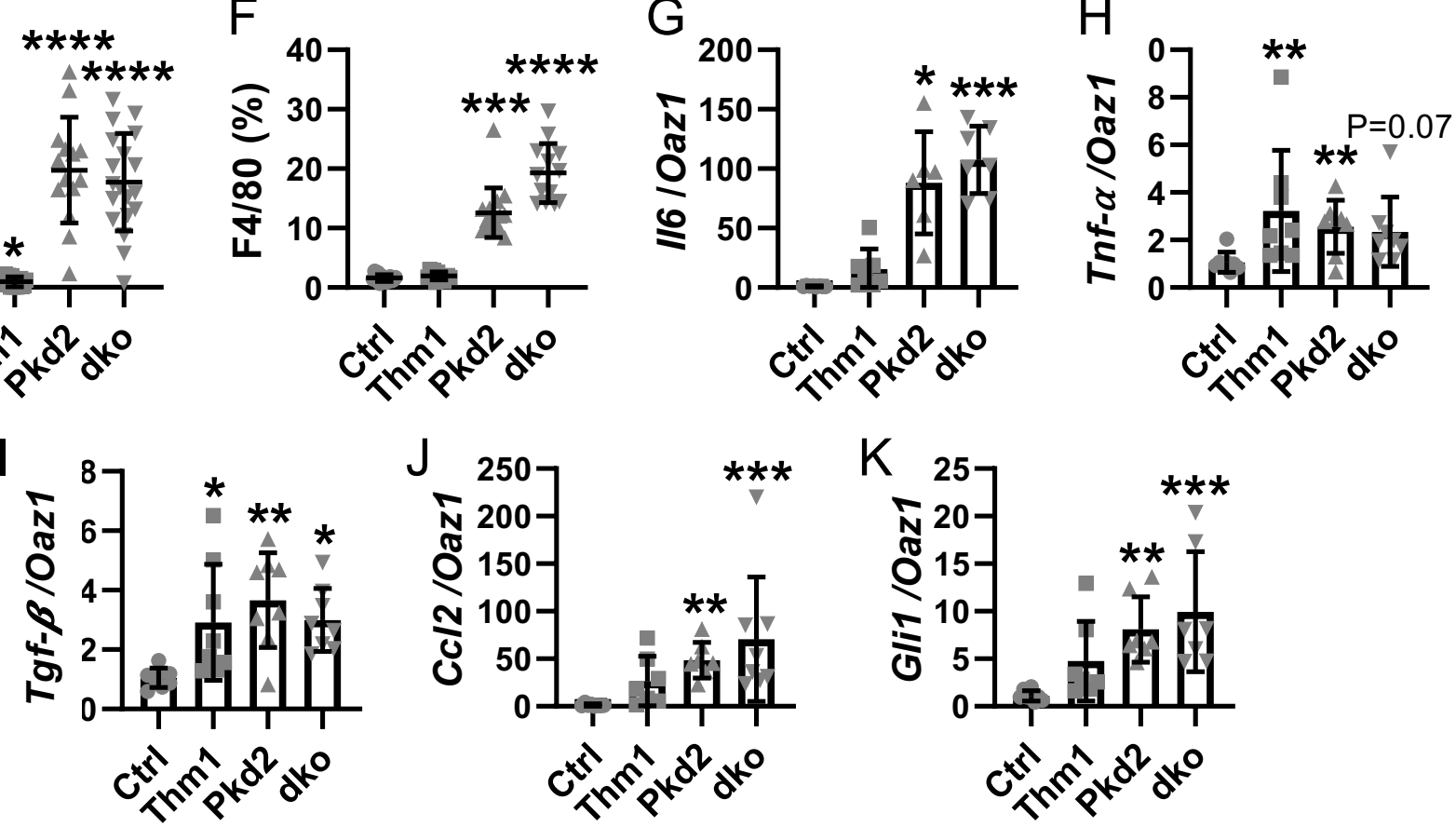

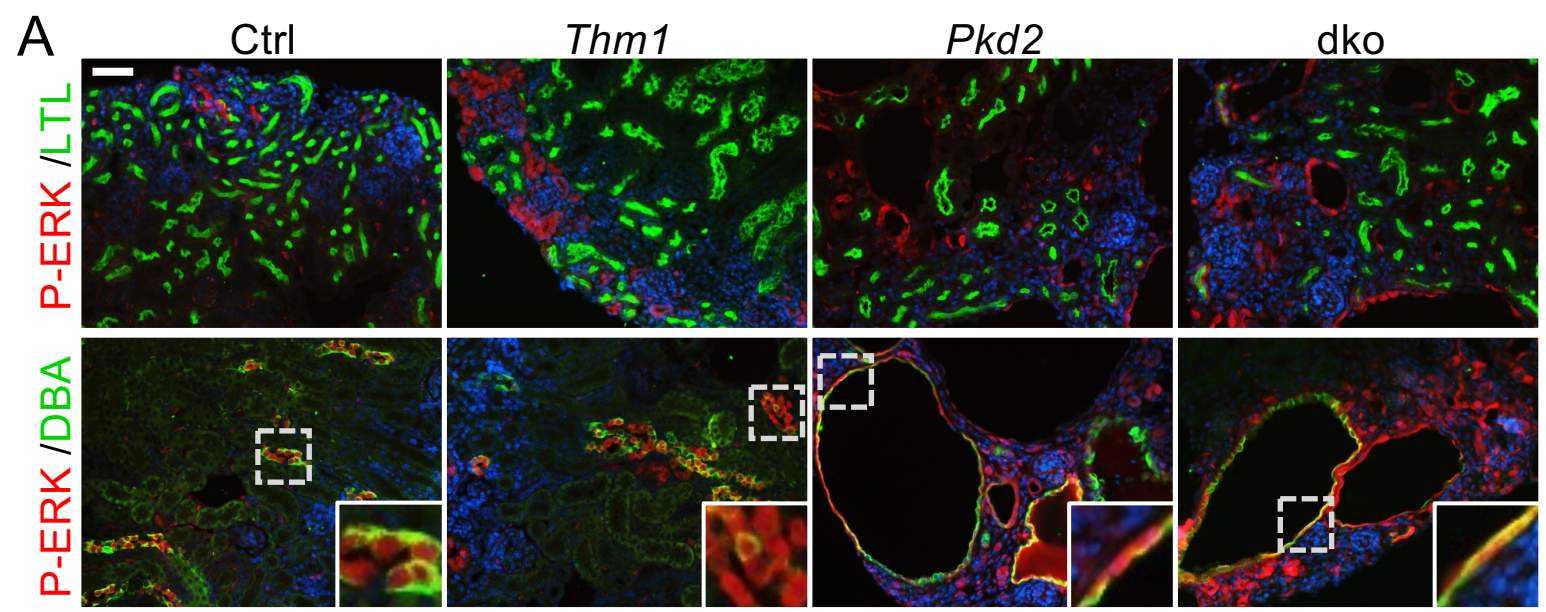

B

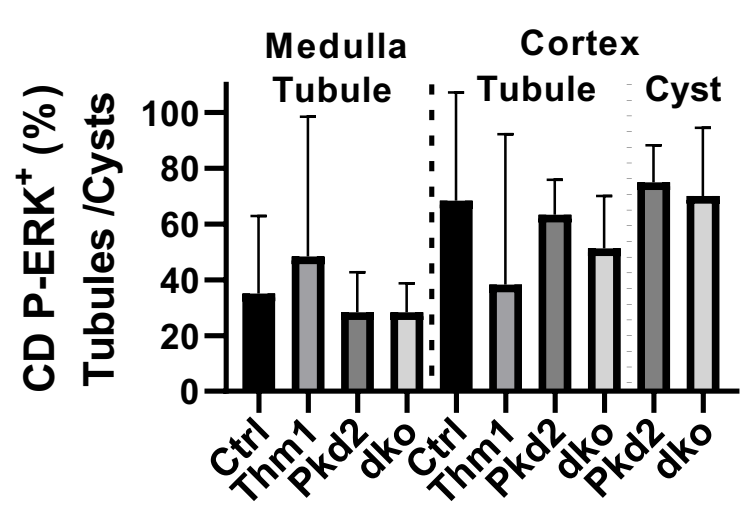

C

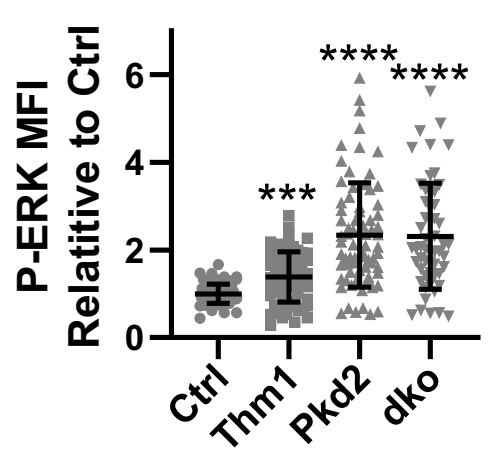

D

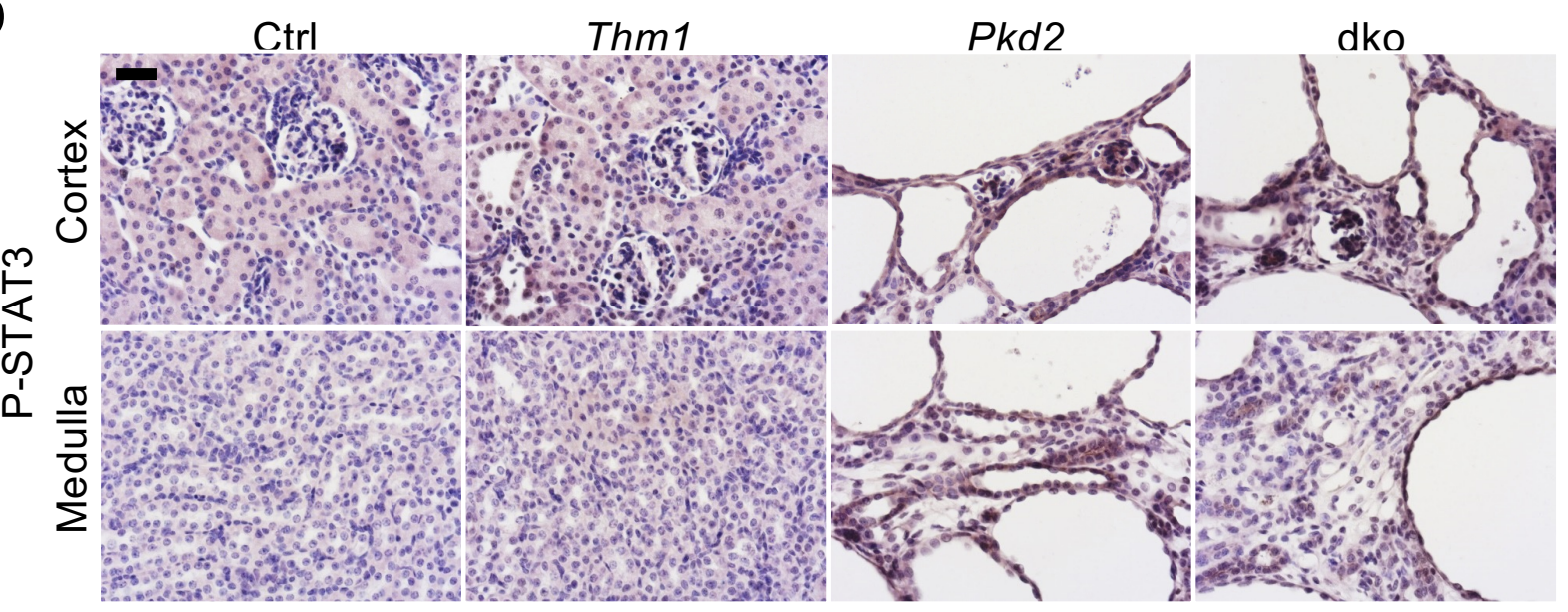

E

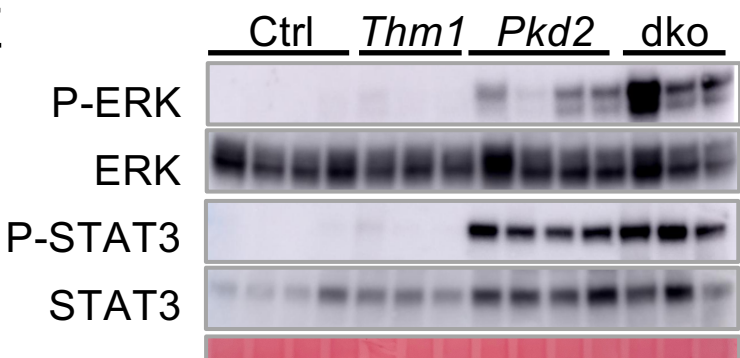

Ponceau S

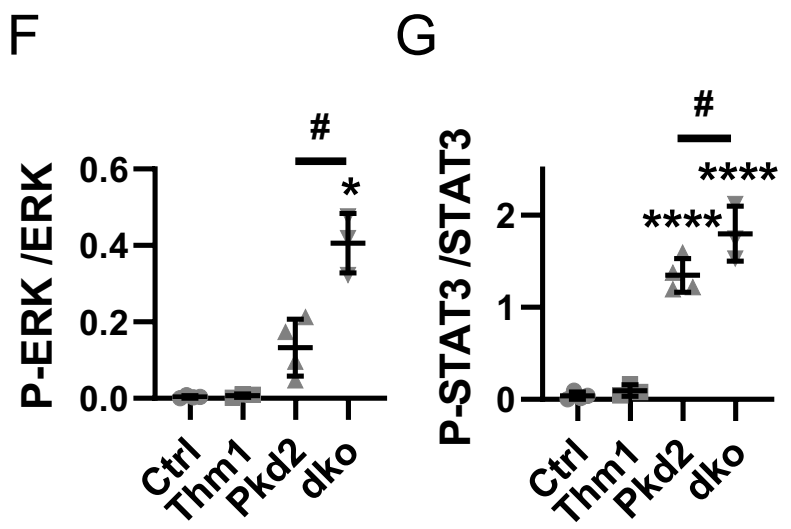



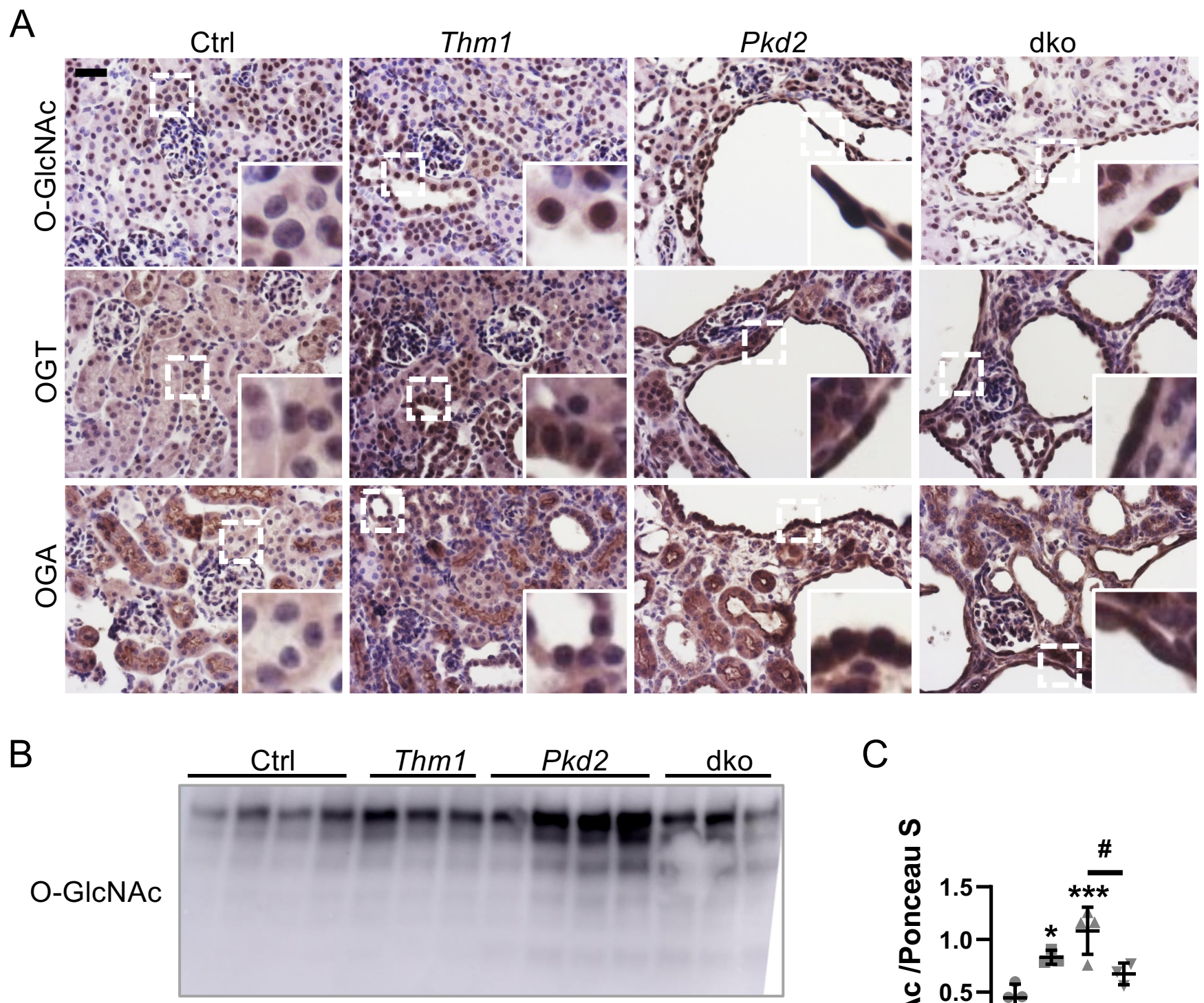

Ponceau S

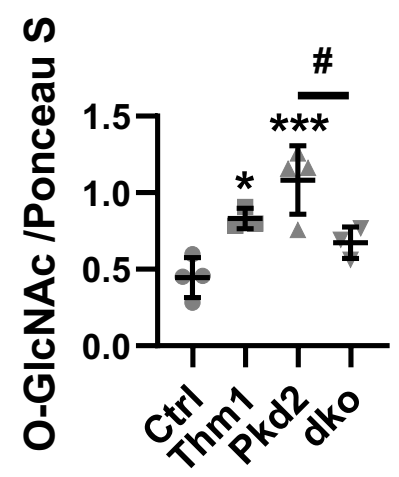



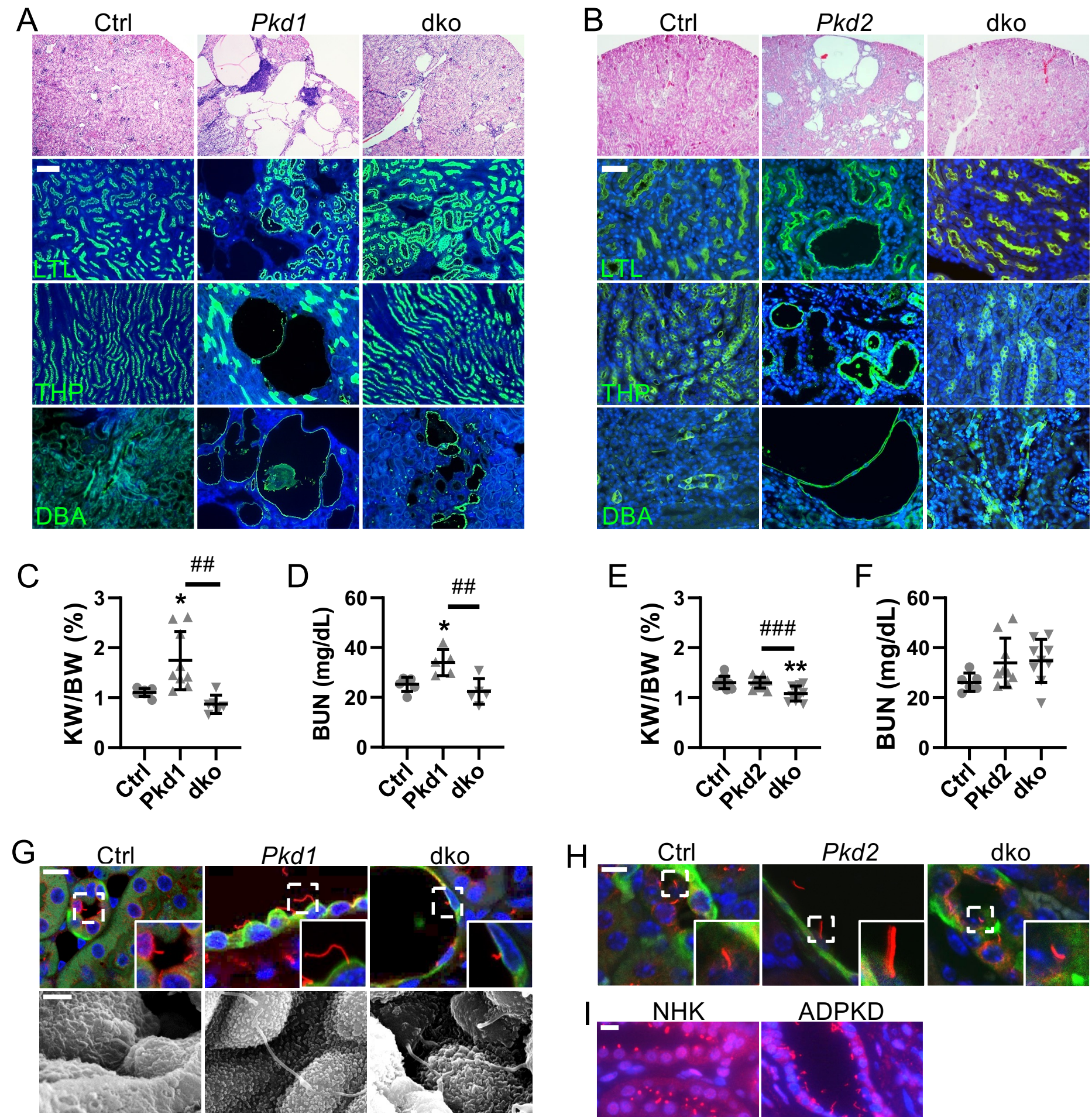
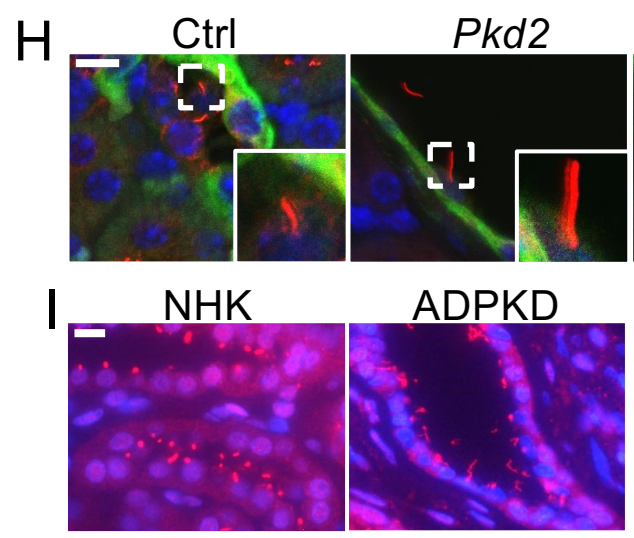
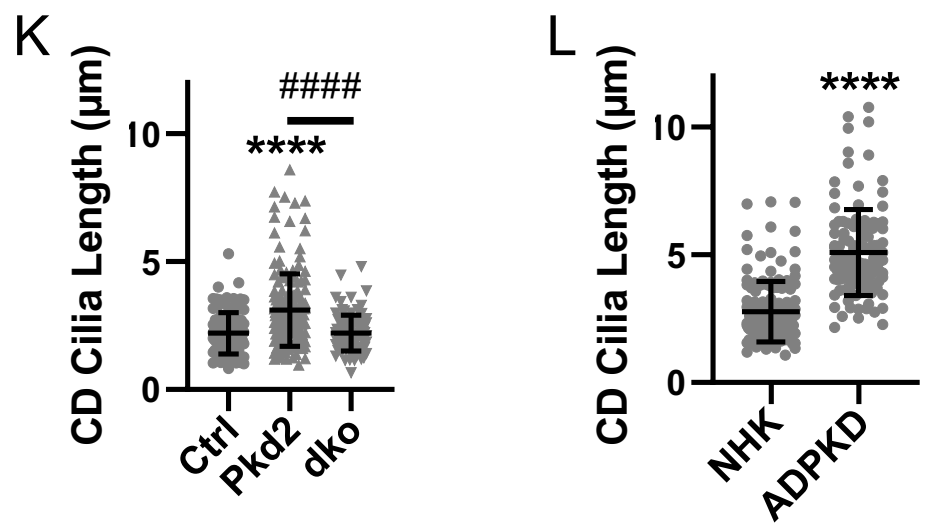

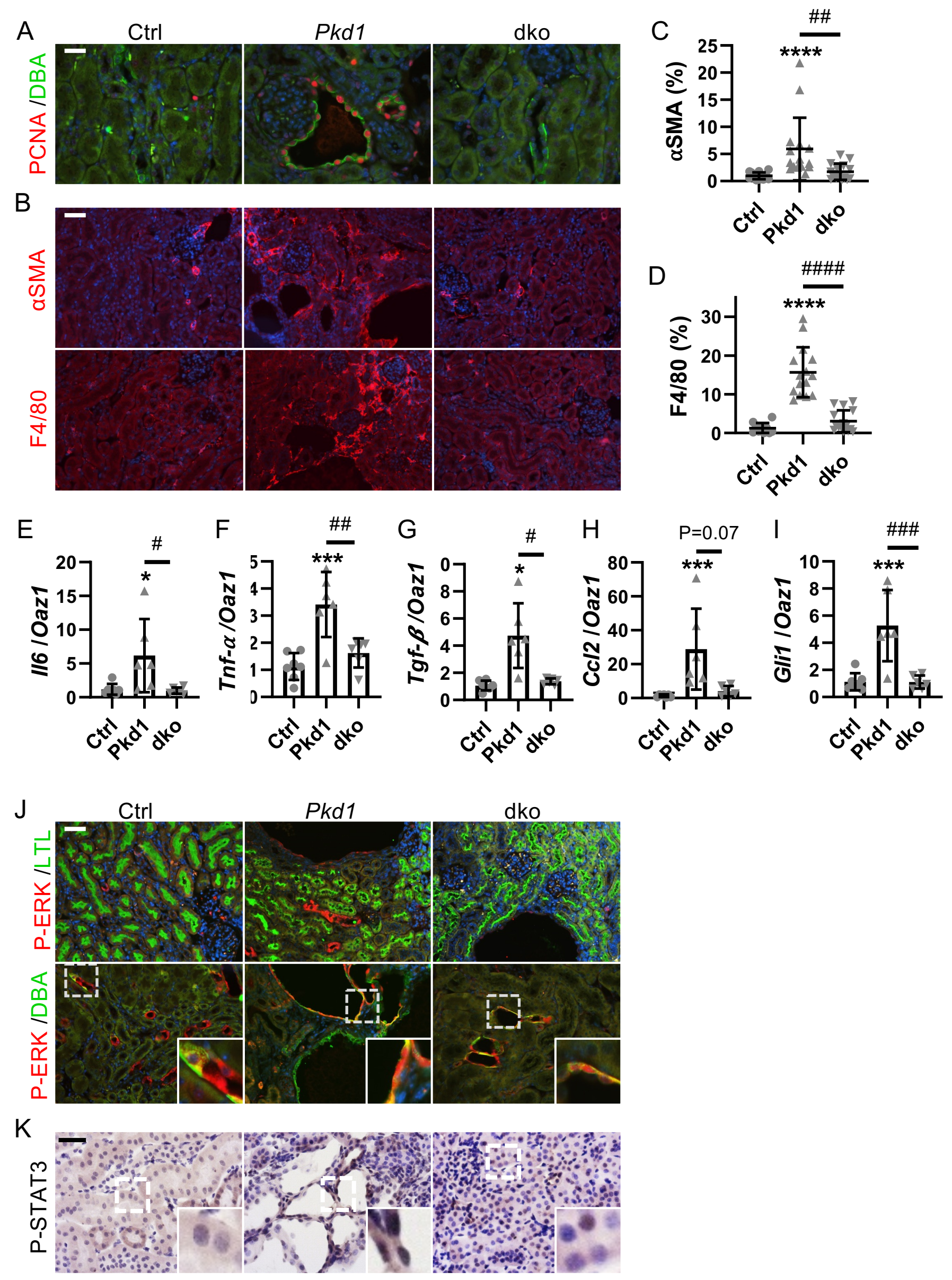
A

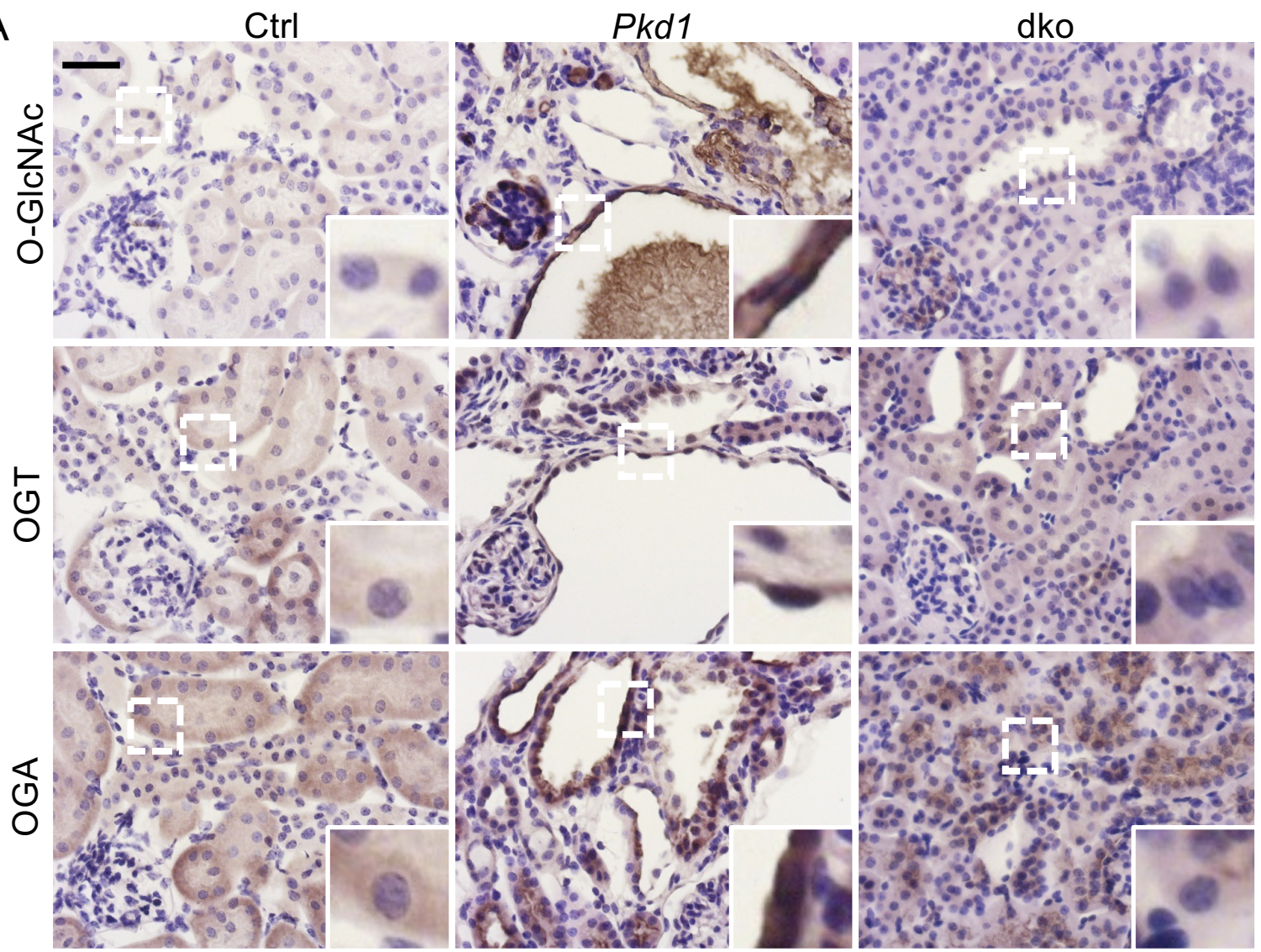

B

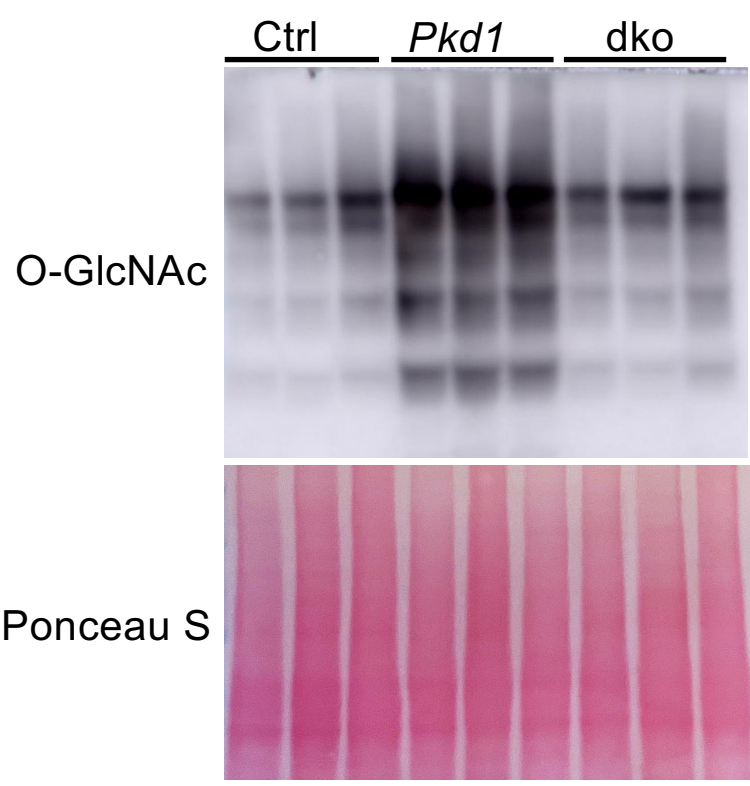

C

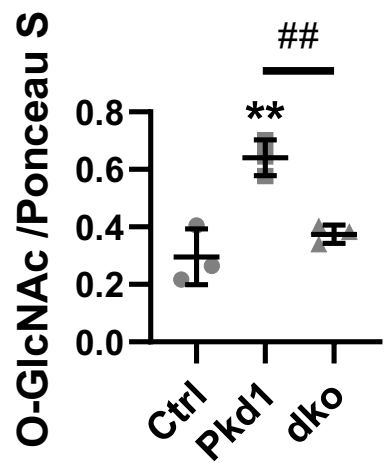




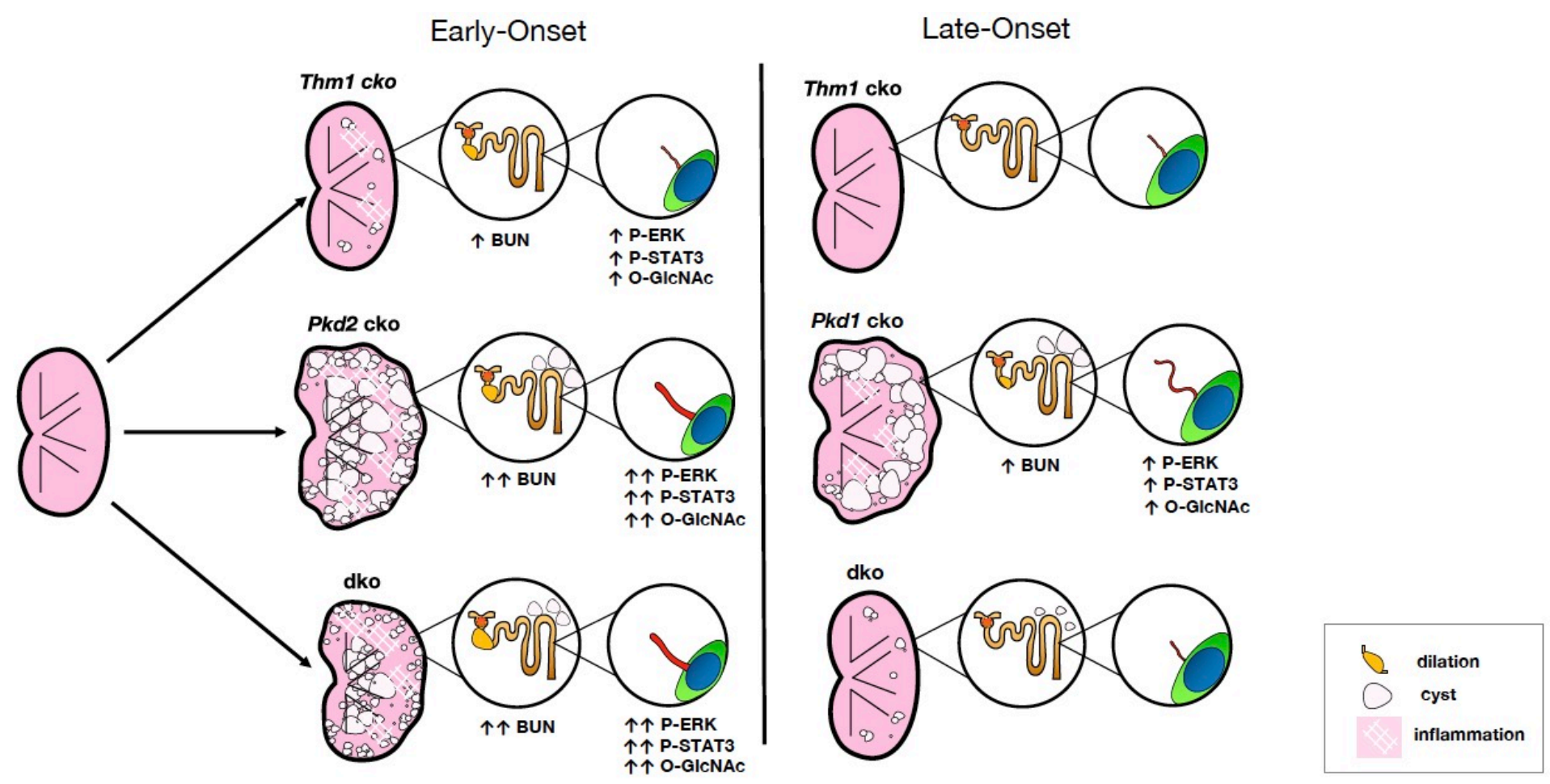

